\title{
Planar four node piezoelectric elements with drilling degrees of freedom
}

\author{
C. S. Long ${ }^{1}$, P. W. Loveday ${ }^{2}$ and Albert A. Groenwold ${ }^{1, *, \dagger}$ \\ ${ }^{1}$ Department of Mechanical Engineering, University of Pretoria, Pretoria 0002, South Africa \\ ${ }^{2}$ Centre for Integrated Sensing Systems, CSIR, P.O. Box 395, Pretoria 0001, South Africa
}

\begin{abstract}
SUMMARY
Several new planar four node piezoelectric elements with drilling degrees of freedom are presented. We begin by deriving two families of variational formulations accounting for piezoelectricity and in-plane rotations. The first family retains the skew-symmetric part of the stress tensor, while in the second, the skew part of stress is eliminated from the functional. The finite elements derived from two of the variational formulations derived in this paper are investigated. The first element is based on an 'irreducible' form, while the other is based on a fully mixed functional, with both stress and electric flux density assumed. Our new elements are shown to be accurate and robust in comparison with a number of existing elements, for several benchmark test problems. Copyright (C) 2005 John Wiley \& Sons, Ltd.
\end{abstract}

KEY WORDS: piezoelectricity; drilling degrees of freedom; mixed variational formulation; finite element

\section{INTRODUCTION}

In recent years, the use of smart materials has become widespread and almost commonplace. The technology employed in piezoelectric applications in particular, has reached a mature level, and piezoelectric materials are frequently used in engineering applications.

Piezoelectric materials transfer electric energy to mechanical energy and vice versa, and can therefore be used as either actuators or sensors, or both. Applications include ultrasonic transducers for sonar and medical purposes, compact piezoelectric motors, structural monitoring and/or active damping elements, and even ignition systems.

\footnotetext{
*Correspondence to: Albert A. Groenwold, Department of Mechanical Engineering, University of Pretoria, Pretoria 0002, South Africa.

†E-mail: albert.groenwold@eng.up.ac.za

Contract/grant sponsor: South African NRF THRIP; contract/grant number: 2769
}

Received 31 March 2005

Revised 19 July 2005

Copyright (c) 2005 John Wiley \& Sons, Ltd.

Accepted 1 September 2005 
Analytical closed-form solutions to problems involving piezoelectric materials are often difficult to compute, unless geometries and boundary conditions are relatively simple, see for example References [1,2]. A general numerical method for the solution of piezoelectric problems is therefore essential. Analytical solutions are, however, very useful as benchmark problems.

The finite element method has become a standard modelling utility for various physical processes, including piezoelectricity. Development of piezoelectric finite elements has progressed significantly since the early paper of Allik and Hughes [3]. In fact, Benjeddou [4] presented a survey article in which over 100 recent papers from the open literature are reviewed, indicating the research interest in the field.

The original implementation of Allik and Hughes, and many of the finite elements since, (see Benjeddou for examples), have been based on formulations interpolating for only kinematiclike variables, i.e. displacement and electric potential. These elements are often stiff, inaccurate and sensitive to mesh distortion. To alleviate these problems, mixed and hybrid variational formulations have been developed (see for example Reference [5]), with original contributions in variational formulations for piezoelectric media credited to EerNisse and Holland [6,7]. Various hybrid and mixed finite elements have since been developed, with notable contributions by Cannarozzi and Ubertini [8] and Sze and co-workers [9-13].

Independent of the development of piezoelectric finite elements, many advances have been made in the development of elastic finite elements. One of the significant contributions has been the addition of in-plane rotations, or drilling degrees of freedom (dof's). Drilling dof's are particularly important in shell elements, since the result is a shell element with six dof's per node, which allows for the modelling of beam-slab connections and folded plates.

After several attempts to develop membrane elements with drilling dof's failed, Allman [14] and Bergan and Felippa [15] achieved a previously unattained level of success. Instead of the cubic functions previously used, they employed a quadratic displacement function for the normal component of displacement. Since then, many papers on the subject have appeared, notably those by Jetteur, Jaamei and Frey [16-19] and by Taylor and Simo et al. [20-22]. However, these elements all suffer from the serious drawback that they are rank deficient.

These issues were addressed by Hughes and Brezzi [23], who presented a rigorous mathematical framework in which to formulate elements with independent rotational interpolations. They argue that, utilizing the formulation of Reissner [24], formulations employing 'convenient' displacement, rotation and stress interpolations are doomed to failure. Instead, they propose a modified variational principle, with improved stability properties in the discrete form.

Finite element implementations employing the formulation of Hughes and Brezzi were finally presented by Hughes et al. [25] and Ibrahimbegovic et al. [26, 27]. Since then, the development of membrane finite elements with drilling dof's has been significant. Sze and co-workers $[28,29]$ developed elements with in-plane rotations and assumed stress fields. Groenwold and Stander $[30,31]$ applied the five-point quadrature presented by Dovey [32] to drilling degree of freedom (dof) membranes, which improved the element behaviour through the introduction of a 'soft' higher order deformation mode. Later Geyer and Groenwold [33] also developed assumed stress finite elements with drilling dof's.

The aim of this paper is to combine the theory, developed for elastic elements with drilling dof's, with some recent advances in piezoelectric finite element technology. The result is two new families of accurate, piezoelectric finite elements with drilling dof's. 
We endeavour to, not only improve on element accuracy, but importantly, improve on the modelling capabilities of existing piezoelectric finite elements. In fact, the piezoelectric elements developed herein are currently being used in a topology optimization environment, together with elastic elements possessing drilling dof's [34]. Their accuracy can also be exploited in fracture analyses. Aside from the improved accuracy, these elements can be employed to calculate through-thickness phenomena in thick piezoelectric shells. The variational formulations can also be used to generate three-dimensional solid elements with drilling dof's. These solid elements would possess three displacement, three rotational and one potential dof per node.

Our paper is set out as follows. In Section 2, the equations governing the linear electroelastic problem are presented. Section 3 introduces a number of variational formulations accounting for piezoelectricity and in-plane rotations. In Section 4 the interpolations used in the finite element implementations are given. Section 5 details the finite element implementation of our variational formulations. Section 6 contains the results of a numerical evaluation of our new elements. Finally, in Section 7 some closing remarks are communicated.

\section{GOVERNING EQUATIONS}

In this section, the equations governing the linear electroelastic problem are presented in strong form. Let $\bar{\Omega}$ be a closed and bounded domain occupied by a body in three-dimensional space. The interior part of $\bar{\Omega}$ is denoted by $\Omega$ and its boundary by $\partial \Omega, \Omega \cup \partial \Omega=\bar{\Omega}$. The measure of $\Omega$ is $V$ and the measure of $\partial \Omega$ is $S$. $\mathscr{V}$ is the vector space associated with the Euclidean point space and $\mathscr{L}$ the space of all linear applications of $\mathscr{V}$ into $\mathscr{V}$, which possesses inner product $\mathbf{A} \cdot \mathbf{B}=\operatorname{tr}\left(\mathbf{A}^{\mathrm{t}} \mathbf{B}\right), \quad \mathbf{A}, \mathbf{B} \in \mathscr{L}$ and $\mathbf{A}^{\mathrm{t}}$ the transpose of $\mathbf{A}$ (see Reference [35]). Reference will also be made to subsets of $\mathscr{L}$, namely $\mathcal{S}$ and $\mathscr{W}$ which contain, respectively symmetric and skew-symmetric tensors in $\mathscr{L}$.

The boundary $\partial \Omega$, is split into four parts, $\partial \Omega_{u}, \partial \Omega_{t}, \partial \Omega_{\phi}$ and $\partial \Omega_{d}$ such that $\partial \Omega_{u} \cup \partial \Omega_{t}=\partial \Omega_{\phi} \cup$ $\partial \boldsymbol{\Omega}_{d}=\partial \boldsymbol{\Omega}$ and $\partial \boldsymbol{\Omega}_{u} \cap \partial \boldsymbol{\Omega}_{t}=\partial \boldsymbol{\Omega}_{\phi} \cap \partial \boldsymbol{\Omega}_{d}=\emptyset$. On $\partial \boldsymbol{\Omega}_{u}$ displacements $\overline{\mathbf{u}}$ are prescribed, while on $\partial \Omega_{t}$ the traction $\overline{\mathbf{t}}$ is prescribed. Similarly, on $\partial \boldsymbol{\Omega}_{\phi}$ the prescribed potentials are $\bar{\phi}$ and on $\partial \Omega_{d}$ the density of the electric charge $\bar{d}$ is prescribed.

The Euclidean decomposition of second-rank tensors is frequently employed, e.g.

$$
\mathbf{T}=\operatorname{symm} \mathbf{T}+\operatorname{skew} \mathbf{T}
$$

where

$$
\begin{array}{r}
\text { symm } \mathbf{T}=\frac{1}{2}\left(\mathbf{T}+\mathbf{T}^{\mathrm{t}}\right) \\
\text { skew } \mathbf{T}=\frac{1}{2}\left(\mathbf{T}-\mathbf{T}^{\mathrm{t}}\right)
\end{array}
$$

The linear electroelastic problem is governed by the following conditions at all points $\mathbf{x} \in \bar{\Omega}$.

\subsection{Constitutive equations}

There exist four equivalent versions of the electroelastic constitutive equations, depending on the choice of independent variables (see for example Reference [36]). The constitutive equations, 
in terms of strain and electric field are:

$$
\begin{aligned}
& \mathbf{T}=\mathbf{c}_{E} \mathbf{S}-\mathbf{e}^{\mathrm{t}} \mathbf{E} \\
& \mathbf{D}=\mathbf{e} \mathbf{S}+\boldsymbol{\varepsilon}_{S} \mathbf{E}
\end{aligned}
$$

where $\mathbf{T}$ and $\mathbf{S}$ are the stress and strain tensors, $\mathbf{D}$ is the electric flux density, also referred to as electric displacement (see for example Reference [10]), and $\mathbf{E}$ denotes the electric field. Furthermore, $\mathbf{c}_{E}$ is a fourth-order tensor of elastic stiffness, measured at constant electric field as indicated by the subscript ' $E$ '. $\boldsymbol{\varepsilon}_{S}$ is the second-order permittivity tensor at constant strain, and $\mathbf{e}$ is a third-order electroelastic, or piezoelectric, coupling tensor. Both $\mathbf{c}_{E}$ and $\boldsymbol{\varepsilon}_{S}$ are symmetric and positive definite. As described by Cannarozzi and Ubertini [8], e is such that the product $\mathbf{e}^{\mathbf{t}} \mathbf{a}$ is a second-order symmetric tensor for each vector $\mathbf{a}$, with $\mathbf{e}^{\mathrm{t}}$ defined as $\mathbf{A} \cdot \mathbf{e}^{\mathbf{t}} \mathbf{a}=\mathbf{e A} \cdot \mathbf{a}$, and $\mathbf{A}$ a symmetric second-order tensor. Incidentally, the constitutive relations in terms of strain and electric field were used in the original finite element implementations [3].

Since the derivation of the other forms of the constitutive equations, through Legendre transformation, are well known (see for example References $[8,36]$ ) they will simply be stated here without further elaboration.

In terms of strain $\mathbf{S}$ and electric flux density $\mathbf{D}$, the relations are

$$
\begin{aligned}
& \mathbf{T}=\mathbf{c}_{D} \mathbf{S}-\mathbf{h}^{\mathrm{t}} \mathbf{D} \\
& \mathbf{E}=-\mathbf{h S}+\chi_{S} \mathbf{D}
\end{aligned}
$$

and the constitutive terms are computed as

$$
\mathbf{c}_{D}=\mathbf{c}_{E}+\mathbf{e}^{\mathrm{t}} \boldsymbol{\varepsilon}_{S}^{-1} \mathbf{e}, \quad \mathbf{h}=\boldsymbol{\varepsilon}_{S}^{-1} \mathbf{e}, \quad \chi_{S}=\boldsymbol{\varepsilon}_{S}^{-1}
$$

Rewriting in terms of stress $\mathbf{T}$ and electric field $\mathbf{E}$, we get

$$
\begin{aligned}
& \mathbf{S}=\mathbf{s}_{E} \mathbf{T}+\mathbf{d}^{\mathrm{t}} \mathbf{E} \\
& \mathbf{D}=\mathbf{d} \mathbf{T}+\boldsymbol{\varepsilon}_{T} \mathbf{E}
\end{aligned}
$$

with

$$
\mathbf{s}_{E}=\mathbf{c}_{E}^{-1}, \quad \mathbf{d}=\mathbf{e c}_{E}^{-1}, \quad \boldsymbol{\varepsilon}_{T}=\boldsymbol{\varepsilon}_{S}+\mathbf{e c}_{E}^{-1} \mathbf{e}^{\mathrm{t}}
$$

Finally, with stress $\mathbf{T}$ and electric flux density $\mathbf{D}$ selected as independent variables, we have

$$
\begin{aligned}
& \mathbf{S}=\mathbf{s}_{D} \mathbf{T}+\mathbf{g}^{\mathbf{t}} \mathbf{D} \\
& \mathbf{E}=-\mathbf{g} \mathbf{T}+\chi_{T} \mathbf{D}
\end{aligned}
$$

with

$$
\mathbf{s}_{D}=\left(\mathbf{c}_{E}+\mathbf{e}^{\mathrm{t}} \boldsymbol{\varepsilon}_{S}^{-1} \mathbf{e}\right)^{-1}, \quad \mathbf{g}=\boldsymbol{\varepsilon}_{S}^{-1} \mathbf{e s}_{D}, \quad \chi_{T}=\boldsymbol{\varepsilon}_{S}^{-1}-\boldsymbol{\varepsilon}_{S}^{-1} \mathbf{e s}_{D} \mathbf{e}^{\mathrm{t}} \boldsymbol{\varepsilon}_{S}^{-1}
$$

The tensors $\mathbf{c}_{D}, \mathbf{s}_{E}, \mathbf{s}_{D}, \chi_{S}, \chi_{T}$ and $\boldsymbol{\varepsilon}_{T}$ are all symmetric and positive definite, and $\mathbf{h}, \mathbf{d}$ and $\mathbf{g}$ are third-order tensors with the same properties as $\mathbf{e}$. In the presentation of the constitutive equations we have used a condensed notation, assuming symmetric stress and strain tensors for 
the sake of clarity. That is to say, in (4), (5), (7) and (9), $\mathbf{T} \equiv \operatorname{symm} \mathbf{T}$ and $\mathbf{S} \equiv \operatorname{symm} \mathbf{S}$. This is of importance, since the stress tensor in the formulations to follow are not a priori assumed to be symmetric.

\subsection{Compatibility conditions}

The strain-displacement and electric field-potential relationships, together with the displacement and electric potential boundary conditions are, respectively

$$
\begin{aligned}
& \mathbf{S}=\operatorname{symm} \nabla \mathbf{u} \quad \text { in } \Omega \\
& \mathbf{E}=-\nabla \phi \quad \text { in } \Omega \\
& \mathbf{u}=\overline{\mathbf{u}} \quad \text { on } \partial \Omega_{u} \\
& \phi=\bar{\phi} \quad \text { on } \partial \Omega_{\phi}
\end{aligned}
$$

where $\mathbf{u}$ is the displacement vector field, and $\phi$ represents the scalar electric potential field.

\subsection{Equilibrium conditions}

The static force equilibrium equations and Gauss's Law in differential form, together with the boundary conditions, are given by

$$
\begin{aligned}
\operatorname{div} \mathbf{T}+\mathbf{f}=\mathbf{0} & \text { in } \Omega \\
\operatorname{div} \mathbf{D}-q=0 & \text { in } \Omega \\
\operatorname{symm} \mathbf{T n}=\overline{\mathbf{t}} & \text { on } \partial \Omega_{t} \\
\mathbf{D} \cdot \mathbf{n}=-\bar{d} & \text { on } \partial \Omega_{d}
\end{aligned}
$$

where $\mathbf{f}$ is a distributed body force, $q$ is a distributed electric charge in $\Omega$, and $\mathbf{n}$ is the unit outward normal vector on $\partial \Omega$. Usually $q$ is taken as zero [5], but for completeness it will be included in the presentation to follow.

\subsection{Stress symmetry condition and definition of infinitesimal rotation}

In the current formulation, the stress tensor $\mathbf{T}$ is not a priori assumed to be symmetric, and in-plane rotations are included. The following two additional conditions need to be satisfied:

$$
\begin{aligned}
\text { skew } \mathbf{T} & =\mathbf{0} \quad \text { in } \Omega \\
\psi & =\text { skew } \nabla \mathbf{u} \quad \text { in } \Omega
\end{aligned}
$$

where (19) represents the symmetry condition for stress and (20) is the definition of infinitesimal rotations in terms of displacement gradient. 


\section{VARIATIONAL FORMULATION}

Hughes and Brezzi [23] presented a general framework to construct variational formulations for problems which include rotational freedom. The most general type is their Hu-Washizulike variational formulation accounting for rotations and non-symmetric stress tensors. We now generalize the variational framework of Hughes and Brezzi to account for the piezoelectric effect.

In the functionals to follow, unless otherwise stated, $\mathbf{u}, \phi, \mathbf{E}$ and $\mathbf{D}$ are the displacement, electric potential, electric field and electric flux density fields, respectively. The non-symmetric stress tensor is denoted $\mathbf{T} \in \mathscr{L}$. The skew-symmetric infinitesimal spin or rotation tensor is $\psi \in \mathscr{W}$, and $\mathbf{S} \in \mathcal{S}$ is the symmetric strain tensor. Where applicable, they are sufficiently regular and square integrable functions of $\mathbf{x}$.

\subsection{Hu-Washizu-like variational formulations}

We propose a $\mathrm{Hu}$-Washizu-like variational formulation. We also show how this leads to a Hellinger-Reissner-like functional, as well as functionals in an irreducible form. We do not aim to present a rigorous mathematical study of the presented formulations. Rather, in the second part of this paper, a numerical study of the discrete finite element implementation is presented.

Two formulation families are proposed. The first, designated here as M-Type, retains the skew-symmetric part of the stress tensor. Since part of the stress tensor is always retained, even in its simplest or irreducible form, M-Type functionals will result in a mixed formulation. In the second family, denoted K-Type, the skew-symmetric part of stress is eliminated. The result is an irreducible form which requires only kinematic-like interpolations, i.e. displacement and potential.

3.1.1. M-Type formulation based on functional $\Pi_{\mathrm{M}}^{\mathrm{H}}$. We propose the Hu-Washizu-like functional

$$
\begin{aligned}
& \Pi_{\mathrm{M}}^{\mathrm{H}}(\mathbf{u}, \boldsymbol{\psi}, \mathbf{T}, \mathbf{S}, \phi, \mathbf{D}, \mathbf{E}) \\
& =\frac{1}{2} \int_{\Omega} \mathbf{c}_{E} \mathbf{S} \cdot \mathbf{S} \mathrm{d} V-\int_{\Omega} \mathbf{e S} \cdot \mathbf{E} \mathrm{d} V-\frac{1}{2} \int_{\Omega} \boldsymbol{\varepsilon}_{S} \mathbf{E} \cdot \mathbf{E} \mathrm{d} V \\
& \quad+\int_{\Omega}(\operatorname{symm} \nabla \mathbf{u}-\mathbf{S}) \cdot \operatorname{symm} \mathbf{T} \mathrm{d} V+\int_{\Omega}(\text { skew } \nabla \mathbf{u}-\boldsymbol{\psi}) \cdot \text { skew } \mathbf{T} \mathrm{d} V \\
& \quad+\int_{\Omega}(\nabla \phi+\mathbf{E}) \cdot \mathbf{D} \mathrm{d} V-\frac{1}{2} \gamma^{-1} \int_{\Omega} \mid \text { skew }\left.\mathbf{T}\right|^{2} \mathrm{~d} V-\int_{\Omega} \mathbf{f} \cdot \mathbf{u} \mathrm{d} V+\int_{\Omega} q \phi \mathrm{d} V \\
& \quad-\int_{\partial \Omega_{t}} \overline{\mathbf{t}} \cdot \mathbf{u} \mathrm{d} \mathrm{S}+\int_{\partial \Omega_{d}} \bar{d} \phi \mathrm{d} \mathrm{S}-\int_{\partial \Omega_{u}}(\mathbf{u}-\overline{\mathbf{u}}) \cdot(\mathbf{T n}) \mathrm{d} S-\int_{\partial \Omega_{\phi}}(\phi-\bar{\phi})(\mathbf{D} \cdot \mathbf{n}) \mathrm{d} S
\end{aligned}
$$

where the subscript ' $M$ ' emphasizes that the functional is of M-Type and the superscript ' $H$ ' that it is a Hu-Washizu-like functional. The term $\frac{1}{2} \gamma^{-1} \int_{\Omega} \mid$ skew $\left.\mathbf{T}\right|^{2} \mathrm{~d} V$ was shown to preserve the ellipticity of the discrete problem in linear elastostatics [23]. Recalling the Gauss-Green 
identities, given by

$$
\begin{gathered}
\int_{\Omega} \mathbf{u} \cdot \operatorname{div} \mathbf{T} \mathrm{d} V=-\int_{\Omega} \mathbf{T} \cdot \nabla \mathbf{u} \mathrm{d} V+\int_{\partial \Omega} \mathbf{u} \cdot \mathbf{T n} \mathrm{d} S \\
\int_{\Omega} \phi \operatorname{div} \mathbf{D} \mathrm{d} V=-\int_{\Omega} \mathbf{D} \cdot \nabla \phi \mathrm{d} V+\int_{\partial \Omega} \phi \mathbf{D} \cdot \mathbf{n} \mathrm{d} S
\end{gathered}
$$

and employing the condition of stationarity gives rise to the following variational equation:

$$
\begin{aligned}
0= & \delta \Pi_{\mathrm{M}}^{\mathrm{H}}=\int_{\Omega}\left(\mathbf{c}_{E} \mathbf{S}-\mathbf{e}^{\mathrm{t}} \mathbf{E}-\operatorname{symm} \mathbf{T}\right) \cdot \delta \mathbf{S} \mathrm{d} V-\int_{\Omega}\left(\mathbf{e S}+\boldsymbol{\varepsilon}_{S} \mathbf{E}-\mathbf{D}\right) \cdot \delta \mathbf{E} \mathrm{d} V \\
& +\int_{\Omega}(\operatorname{symm} \nabla \mathbf{u}-\mathbf{S}) \cdot \operatorname{symm} \delta \mathbf{T} \mathrm{d} V+\int_{\Omega}\left(\operatorname{skew} \nabla \mathbf{u}-\boldsymbol{\psi}-\gamma^{-1} \text { skew } \mathbf{T}\right) \cdot \text { skew } \delta \mathbf{T} \mathrm{d} V \\
& -\int_{\Omega} \operatorname{skew~} \mathbf{T} \cdot \delta \boldsymbol{\psi} \mathrm{d} V+\int_{\Omega}(\nabla \phi+\mathbf{E}) \cdot \delta \mathbf{D} \mathrm{d} V-\int_{\Omega}(\operatorname{div} \mathbf{T}+\mathbf{f}) \cdot \delta \mathbf{u} \mathrm{d} V \\
& -\int_{\Omega}(\operatorname{div} \mathbf{D}-q) \delta \phi \mathrm{d} V+\int_{\partial \Omega_{t}}(\mathbf{T} \mathbf{n}-\overline{\mathbf{t}}) \cdot \delta \mathbf{u} \mathrm{d} S+\int_{\partial \Omega_{d}}(\mathbf{D} \cdot \mathbf{n}+\bar{d}) \delta \phi \mathrm{d} S \\
& -\int_{\partial \Omega_{u}}(\mathbf{u}-\overline{\mathbf{u}}) \cdot(\delta \mathbf{T n}) \mathrm{d} S-\int_{\partial \Omega_{\phi}}(\phi-\bar{\phi})(\delta \mathbf{D} \cdot \mathbf{n}) \mathrm{d} \mathrm{S}
\end{aligned}
$$

All of the necessary Euler-Lagrange equations appear in (24). The variation $\delta \psi$ enforces skew $\mathbf{T}=\mathbf{0}$ in $\Omega$, while skew $\delta \mathbf{T}$ enforces compatibility between rotations and the skew part of the displacement gradient.

3.1.2. K-Type formulation based on functional $\Pi_{\mathrm{K}}^{\mathrm{H}}$. The skew-symmetric part of the stress tensor can be eliminated using the Euler-Lagrange equation $\gamma^{-1}$ skew $\mathbf{T}=$ skew $\nabla \mathbf{u}-\boldsymbol{\psi}$ which appears in (24) as demonstrated by Hughes and Brezzi [23], to obtain the K-Type Hu-Washizu-like functional:

$$
\begin{aligned}
\Pi_{\mathrm{K}}^{\mathrm{H}}(\mathbf{u}, \boldsymbol{\psi}, \mathbf{T}, \mathbf{S}, \phi, \mathbf{D}, \mathbf{E})= & \frac{1}{2} \int_{\Omega} \mathbf{c}_{E} \mathbf{S} \cdot \mathbf{S} \mathrm{d} V-\int_{\Omega} \mathbf{e} \mathbf{S} \cdot \mathbf{E} \mathrm{d} V \\
& -\frac{1}{2} \int_{\Omega} \boldsymbol{\varepsilon}_{S} \mathbf{E} \cdot \mathbf{E} \mathrm{d} V+\int_{\Omega}(\operatorname{symm} \nabla \mathbf{u}-\mathbf{S}) \cdot \operatorname{symm} \mathbf{T} \mathrm{d} V \\
& +\int_{\Omega}(\nabla \phi+\mathbf{E}) \cdot \mathbf{D} \mathrm{d} V+\frac{1}{2} \gamma \int_{\Omega}|\operatorname{skew} \nabla \mathbf{u}-\boldsymbol{\psi}|^{2} \mathrm{~d} V \\
& -\int_{\Omega} \mathbf{f} \cdot \mathbf{u} \mathrm{d} V-\int_{\Omega} q \phi \mathrm{d} V-\int_{\partial \Omega_{t}} \overline{\mathbf{t}} \cdot \mathbf{u} \mathrm{d} \mathrm{S}+\int_{\partial \Omega_{d}} \bar{d} \phi \mathrm{d} S \\
& -\int_{\partial \Omega_{u}}(\mathbf{u}-\overline{\mathbf{u}}) \cdot(\mathbf{T n}) \mathrm{d} S-\int_{\partial \Omega_{\phi}}(\phi-\bar{\phi})(\mathbf{D} \cdot \mathbf{n}) \mathrm{d} S
\end{aligned}
$$


Taking the first variation, applying the Gauss-Green identities and recognizing $\gamma($ skew $\nabla \mathbf{u}-\boldsymbol{\psi})$ as being skew $\mathbf{T}$, we get

$$
\begin{aligned}
\delta \Pi_{\mathrm{K}}^{\mathrm{H}}= & 0=\int_{\Omega}\left(\mathbf{c}_{E} \mathbf{S}-\mathbf{e}^{\mathrm{t}} \mathbf{E}-\operatorname{symm} \mathbf{T}\right) \cdot \delta \mathbf{S} \mathrm{d} V-\int_{\Omega}\left(\mathbf{e S}+\boldsymbol{\varepsilon}_{S} \mathbf{E}-\mathbf{D}\right) \cdot \delta \mathbf{E} \mathrm{d} V \\
& +\int_{\Omega}(\operatorname{symm} \nabla \mathbf{u}-\mathbf{S}) \cdot \operatorname{symm} \delta \mathbf{T} \mathrm{d} V-\int_{\Omega}(\gamma(\text { skew } \nabla \mathbf{u}-\psi)) \cdot \delta \psi \mathrm{d} V \\
& +\int_{\Omega}(\nabla \phi+\mathbf{E}) \cdot \delta \mathbf{D} \mathrm{d} V-\int_{\Omega}(\operatorname{div} \mathbf{T}+\mathbf{f}) \cdot \delta \mathbf{u d} V-\int_{\Omega}(\operatorname{div} \mathbf{D}-q) \delta \phi \mathrm{d} V \\
& +\int_{\partial \Omega_{t}}(\mathbf{T n}-\overline{\mathbf{t}}) \cdot \delta \mathbf{u} \mathrm{d} \mathrm{S}+\int_{\partial \Omega_{d}}(\mathbf{D} \cdot \mathbf{n}+\bar{d}) \delta \phi \mathrm{d} \mathrm{S} \\
& -\int_{\partial \Omega_{u}}(\mathbf{u}-\overline{\mathbf{u}}) \cdot(\delta \mathbf{T n}) \mathrm{d} S-\int_{\partial \Omega_{\phi}}(\phi-\bar{\phi})(\delta \mathbf{D} \cdot \mathbf{n}) \mathrm{d} S
\end{aligned}
$$

which again contains all the necessary Euler equations. Here, the variation $\delta \psi$ simultaneously enforces skew $\mathbf{T}=\mathbf{0}$ in $\Omega$ and compatibility between rotations and the skew part of displacement gradient.

\subsection{Irreducible formulations}

In order to simplify the formulation for finite element implementation, the irreducible form of $\Pi_{\mathrm{M}}^{\mathrm{H}}$ is now derived.

3.2.1. M-Type formulation based on functional $\Pi_{\mathrm{M}}$. The mechanical and electrical compatibilities (11) and (12), respectively, are enforced a priori. Therefore, $\mathbf{S}=\operatorname{symm} \nabla \mathbf{u}$, and $\mathbf{E}=-\nabla \phi$ are substituted into (21). The functional which results is

$$
\begin{aligned}
\Pi_{\mathrm{M}}(\mathbf{u}, \boldsymbol{\psi}, \text { skew } \mathbf{T}, \phi)= & \frac{1}{2} \int_{\Omega} \mathbf{c}_{E} \operatorname{symm} \nabla \mathbf{u} \cdot \operatorname{symm} \nabla \mathbf{u} \mathrm{d} V+\int_{\Omega} \text { esymm } \nabla \mathbf{u} \cdot \nabla \phi \mathrm{d} V \\
& -\frac{1}{2} \int_{\Omega} \boldsymbol{\varepsilon}_{S} \nabla \phi \cdot \nabla \phi \mathrm{d} V+\int_{\Omega}(\text { skew } \nabla \mathbf{u}-\psi) \cdot \text { skew } \mathbf{T} \mathrm{d} V \\
& -\frac{1}{2} \gamma^{-1} \int_{\Omega}|\operatorname{skew} \mathbf{T}|^{2} \mathrm{~d} V-\int_{\Omega} \mathbf{f} \cdot \mathbf{u} \mathrm{d} V+\int_{\Omega} q \phi \mathrm{d} V \\
& -\int_{\partial \Omega_{t}} \overline{\mathbf{t}} \cdot \mathbf{u} \mathrm{dS}+\int_{\partial \Omega_{d}} \bar{d} \phi \mathrm{dS}
\end{aligned}
$$

where both $\mathbf{u}$ and $\phi$ are admissible, and therefore satisfy the essential boundary conditions. After some algebra, the first variation of $\Pi_{M}$ reduces to

$$
\delta \Pi_{\mathrm{M}}=0=\int_{\Omega}\left(\text { skew } \nabla \mathbf{u}-\psi-\gamma^{-1} \text { skew } \mathbf{T}\right) \cdot \text { skew } \delta \mathbf{T} \mathrm{d} V-\int_{\Omega} \text { skew } \mathbf{T} \cdot \delta \boldsymbol{\psi} \mathrm{d} V
$$




$$
\begin{aligned}
& -\int_{\Omega}(\operatorname{div} \mathbf{T}+\mathbf{f}) \cdot \delta \mathbf{u} \mathrm{d} V-\int_{\Omega}(\operatorname{div} \mathbf{D}-q) \delta \phi \mathrm{d} V+\int_{\partial \Omega_{t}}(\mathbf{T} \mathbf{n}-\overline{\mathbf{t}}) \cdot \delta \mathbf{u} \mathrm{d} S \\
& +\int_{\partial \Omega_{d}}(\mathbf{D} \cdot \mathbf{n}+\bar{d}) \delta \phi \mathrm{d} \mathbf{S}
\end{aligned}
$$

which again contains all the necessary Euler-Lagrange equations.

\subsection{Fully mixed Hellinger-Reissner-like formulations}

Strain $\mathbf{S}$ and electric field $\mathbf{E}$ can be eliminated from the $\mathrm{Hu}-$ Washizu-like functionals by substituting the constitutive relations in (9) into functionals $\Pi_{\mathrm{M}}^{\mathrm{H}}$ and $\Pi_{\mathrm{K}}^{\mathrm{H}}$, resulting in fully mixed Hellinger-Reissner-like functionals.

3.3.1. M-Type formulation based on functional $\Pi_{\mathrm{M}}^{T D}$. We substitute the constitutive relations in terms of stress $\mathbf{T}$ and electric flux density $\mathbf{D}$ into $\Pi_{\mathrm{M}}^{\mathrm{H}}$, and introduce the functional $\Pi_{\mathrm{M}}^{T D}$. The superscripts ' $T$ ' and ' $D$ ' represent the additional independent variables (supplemental to the kinematic variables):

$$
\begin{aligned}
\Pi_{\mathrm{M}}^{T D}(\mathbf{u}, \boldsymbol{\psi}, \mathbf{T}, \phi, \mathbf{D})= & -\frac{1}{2} \int_{\Omega} \mathbf{s}_{D} \operatorname{symm} \mathbf{T} \cdot \operatorname{symm} \mathbf{T} \mathrm{d} V-\int_{\Omega} \mathbf{g} \operatorname{symm} \mathbf{T} \cdot \mathbf{D} \mathrm{d} V \\
& +\frac{1}{2} \int_{\Omega} \chi_{T} \mathbf{D} \cdot \mathbf{D} \mathrm{d} V+\int_{\Omega} \operatorname{symm} \nabla \mathbf{u} \cdot \operatorname{symm} \mathbf{T} \mathrm{d} V+\int_{\Omega} \nabla \phi \cdot \mathbf{D} \mathrm{d} V \\
& +\int_{\Omega}(\operatorname{skew} \nabla \mathbf{u}-\boldsymbol{\psi}) \cdot \operatorname{skew} \mathbf{T} \mathrm{d} V-\frac{1}{2} \gamma^{-1} \int_{\Omega}|\operatorname{skew} \mathbf{T}|^{2} \mathrm{~d} V-\int_{\Omega} \mathbf{f} \cdot \mathbf{u} \mathrm{d} V \\
& +\int_{\Omega} q \phi \mathrm{d} V-\int_{\partial \Omega_{t}} \overline{\mathbf{t}} \cdot \mathbf{u} \mathrm{dS}+\int_{\partial \Omega_{d}} \bar{d} \phi \mathrm{dS}-\int_{\partial \Omega_{u}}(\mathbf{u}-\overline{\mathbf{u}}) \cdot(\mathbf{T n}) \mathrm{dS} \\
& -\int_{\partial \Omega_{\phi}}(\phi-\bar{\phi})(\mathbf{D} \cdot \mathbf{n}) \mathrm{dS}
\end{aligned}
$$

After applying the Gauss-Green identities, the result of the first variation is

$$
\begin{aligned}
\delta \Pi_{\mathrm{M}}^{T D}= & =-\int_{\Omega}\left(\mathbf{s}_{D} \operatorname{symm} \mathbf{T}+\mathbf{g}^{\mathrm{t}} \mathbf{D}-\operatorname{symm} \nabla \mathbf{u}\right) \cdot \operatorname{symm} \delta \mathbf{T} \mathrm{d} V \\
& +\int_{\Omega}\left(\text { skew } \nabla \mathbf{u}-\psi-\gamma^{-1} \text { skew } \mathbf{T}\right) \cdot \text { skew } \delta \mathbf{T} \mathrm{d} V \\
& +\int_{\Omega}\left(-\mathbf{g} \operatorname{symm} \mathbf{T}+\chi_{T} \mathbf{D}+\nabla \phi\right) \cdot \delta \mathbf{D} \mathrm{d} V-\int_{\Omega} \operatorname{skew} \mathbf{T} \cdot \delta \boldsymbol{\psi} \mathrm{d} V
\end{aligned}
$$




$$
\begin{aligned}
& -\int_{\Omega}(\operatorname{div} \mathbf{T}+\mathbf{f}) \cdot \delta \mathbf{u} \mathrm{d} V-\int_{\Omega}(\operatorname{div} \mathbf{D}-q) \delta \phi \mathrm{d} V+\int_{\partial \Omega_{t}}(\mathbf{T} \mathbf{n}-\overline{\mathbf{t}}) \cdot \delta \mathbf{u} \mathrm{d} S \\
& +\int_{\partial \Omega_{d}}(\mathbf{D} \cdot \mathbf{n}+\bar{d}) \delta \phi \mathrm{d} S-\int_{\partial \Omega_{u}}(\mathbf{u}-\overline{\mathbf{u}}) \cdot(\delta \mathbf{T n}) \mathrm{d} S-\int_{\partial \Omega_{\phi}}(\phi-\bar{\phi})(\delta \mathbf{D} \cdot \mathbf{n}) \mathrm{d} S
\end{aligned}
$$

where the Euler equation $\mathbf{s}_{D}$ symm $\mathbf{T}+\mathbf{g}^{\mathrm{t}} \mathbf{D}=$ symm $\nabla \mathbf{u}$ weakly enforces the relationship between strain derived from compatibility conditions (11), and strain based on stress and electric flux density from constitutive equations (9). Similarly, $-\mathbf{g s y m m} \mathbf{T}+\chi_{T} \mathbf{D}=-\nabla \phi$ enforces weakly the relationship between compatibility (12) and constitutive equations (9) for electric field.

\subsection{Degenerate Hellinger-Reissner-like formulations}

It is also possible to derive functionals with only stress $\mathbf{T}$ or electric flux density $\mathbf{D}$ assumed, additionally to $\mathbf{u}$ and $\phi$, using the remaining forms of the constitutive equations. Since only stress or electric flux density is assumed, we will refer to these functionals as degenerate.

For example, if $\mathbf{S}=\operatorname{symm} \nabla \mathbf{u}$ and the second equation in (5) are substituted into $\Pi_{\mathrm{M}}^{\mathrm{H}}$, we get a functional with electric flux density $\mathbf{D}$ assumed, i.e.

$\Pi_{\mathrm{M}}^{D}(\mathbf{u}, \boldsymbol{\psi}$, skew $\mathbf{T}, \phi, \mathbf{D})=\frac{1}{2} \int_{\Omega} \mathbf{c}_{D} \operatorname{symm} \nabla \mathbf{u} \cdot \operatorname{symm} \nabla \mathbf{u} \mathrm{d} V-\int_{\Omega} \mathbf{h s y m m} \nabla \mathbf{u} \cdot \mathbf{D} \mathrm{d} V$

$$
\begin{aligned}
& +\frac{1}{2} \int_{\Omega} \chi_{S} \mathbf{D} \cdot \mathbf{D} \mathrm{d} V+\int_{\Omega}(\text { skew } \nabla \mathbf{u}-\psi) \cdot \text { skew } \mathbf{T} \mathrm{d} V+\int_{\Omega} \nabla \phi \cdot \mathbf{D} \mathrm{d} V \\
& -\frac{1}{2} \gamma^{-1} \int_{\Omega} \mid \text { skew }\left.\mathbf{T}\right|^{2} \mathrm{~d} V-\int_{\Omega} \mathbf{f} \cdot \mathbf{u} \mathrm{d} V+\int_{\Omega} q \phi \mathrm{d} V-\int_{\partial \Omega_{t}} \overline{\mathbf{t}} \cdot \mathbf{u} \mathrm{d} S \\
& +\int_{\partial \Omega_{d}} \bar{d} \phi \mathrm{d} S-\int_{\partial \Omega_{\phi}}(\phi-\bar{\phi})(\mathbf{D} \cdot \mathbf{n}) \mathrm{d} S
\end{aligned}
$$

where $\mathbf{u}$ is admissible.

If on the other hand, we substitute $\mathbf{E}=-\nabla \phi$ and the first equation of (7) into $\Pi_{\mathrm{M}}^{\mathrm{H}}$, a functional with symm $\mathbf{T}$ assumed is achieved, i.e.

$$
\begin{aligned}
\Pi_{\mathrm{M}}^{\mathrm{T}}(\mathbf{u}, \psi, \mathbf{T}, \phi)= & -\frac{1}{2} \int_{\Omega} \mathbf{s}_{E} \operatorname{symm} \mathbf{T} \cdot \operatorname{symm} \mathbf{T} \mathrm{d} V+\int_{\Omega} \mathbf{d} \operatorname{symm} \mathbf{T} \cdot \nabla \phi \mathrm{d} V \\
& -\frac{1}{2} \int_{\Omega} \boldsymbol{\varepsilon}_{T} \nabla \phi \cdot \nabla \phi \mathrm{d} V+\int_{\Omega} \operatorname{symm} \nabla \mathbf{u} \cdot \operatorname{symm} \mathbf{T} \mathrm{d} V \\
& +\int_{\Omega}(\operatorname{skew} \nabla \mathbf{u}-\psi) \cdot \text { skew } \mathbf{T} \mathrm{d} V-\frac{1}{2} \gamma^{-1} \int_{\Omega}|\operatorname{skew} \mathbf{T}|^{2} \mathrm{~d} V
\end{aligned}
$$




$$
\begin{aligned}
& -\int_{\Omega} \mathbf{f} \cdot \mathbf{u} \mathrm{d} V+\int_{\Omega} q \phi \mathrm{d} V-\int_{\partial \Omega_{t}} \overline{\mathbf{t}} \cdot \mathbf{u} \mathrm{d} \mathrm{S} \\
& +\int_{\partial \Omega_{d}} \bar{d} \phi \mathrm{d} \mathrm{S}-\int_{\partial \Omega_{u}}(\mathbf{u}-\overline{\mathbf{u}}) \cdot(\mathbf{T n}) \mathrm{d} \mathrm{S}
\end{aligned}
$$

where $\phi$ is admissible.

The K-Type counterparts of (27), (31) and (32) naturally also exist. They can be constructed by eliminating the skew-symmetric part of the stress tensor similar to the construction of $\Pi_{\mathrm{K}}^{\mathrm{H}}$ from $\Pi_{\mathrm{M}}^{\mathrm{H}}$. For the sake of brevity, the variational formulations based on these functionals are not presented in this paper. They are, however, available from the first author upon request [37]. There is, naturally an alternative formulation with the mechanical part based on the method of incompatible modes $[35,38,39]$, which falls outside the scope of the current study.

\section{FINITE ELEMENT INTERPOLATIONS}

In this section the interpolations, used in the finite element implementation, are briefly discussed. The scalar potential and the independent rotation fields are interpolated as

$$
\begin{aligned}
\phi^{h} & =\sum_{e} \sum_{i=1}^{4} N_{i}^{e}(\xi, \eta) \phi_{i} \\
\psi^{h} & =\sum_{e} \sum_{i=1}^{4} N_{i}^{e}(\xi, \eta) \psi_{i}
\end{aligned}
$$

respectively, with $N_{i}^{e}$ the standard bilinear shape functions. The in-plane displacement approximation is taken as an Allman-type interpolation field, after Ibrahimbegovic et al. [26]

$$
\left\{\begin{array}{l}
u_{1} \\
u_{2}
\end{array}\right\}=\mathbf{u}^{h}=\sum_{e} \sum_{i=1}^{4} N_{i}^{e}(\xi, \eta) \mathbf{u}_{i}+\frac{l_{j k}}{8} \sum_{e} \sum_{i=5}^{8} N S_{i}^{e}(\xi, \eta)\left(\psi_{k}-\psi_{j}\right) \mathbf{n}_{j k}
$$

with $N S_{i}$ the Serendipity shape functions. We do not include a hierarchical bubble shape function. Furthermore, $l_{j k}$ and $\mathbf{n}_{j k}$ denote the length and the outward unit normal vector on the element side associated with the corner nodes $j$ and $k$ (see Figure 1), i.e.

$$
\mathbf{n}_{j k}=\left\{\begin{array}{l}
n_{1} \\
n_{2}
\end{array}\right\}=\left\{\begin{array}{c}
\cos \alpha_{j k} \\
\sin \alpha_{j k}
\end{array}\right\}
$$

and

$$
l_{j k}=\left(\left(x_{k}-x_{j}\right)^{2}+\left(y_{k}-y_{j}\right)^{2}\right)^{1 / 2}
$$

The indices in the above are explicitly given in Appendix A.1.

The skew-symmetric stress field is chosen constant over the element, i.e.

$$
\text { skew } \mathbf{T}^{h}=\sum_{e} T_{0}^{e}
$$




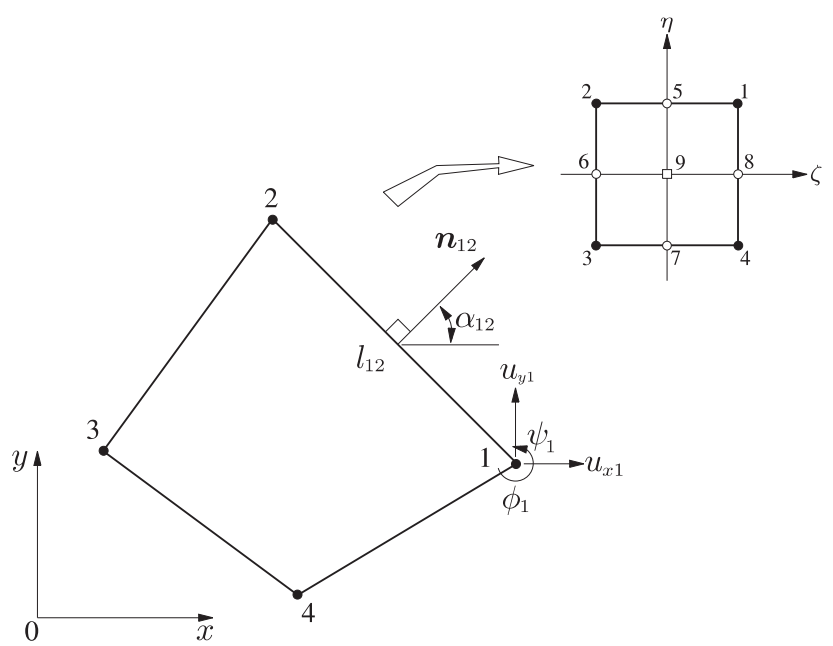

Figure 1. A planar four-node piezoelectric element with drilling rotations.

Using matrix notation, symm $\nabla \mathbf{u}^{e}$ and skew $\nabla \mathbf{u}^{e}$ are, respectively, given by

$$
\operatorname{symm} \nabla \mathbf{u}^{e}=\mathbf{B}_{i}^{e} \mathbf{u}_{i}+\mathbf{G}_{\psi i}^{e} \psi_{i}
$$

and

$$
\text { skew } \nabla \mathbf{u}^{e}=\mathbf{b}_{i}^{e} \mathbf{u}_{i}+\mathbf{g}_{i}^{e} \boldsymbol{\psi}_{i}
$$

The operators arising from this interpolation are also summarized in Appendix A.1. The 'membrane locking correction', due to Taylor [20] is used, i.e. element strains are modified to become

$$
\operatorname{symm} \nabla \tilde{\mathbf{u}}^{e}=\mathbf{B}_{i}^{e} \mathbf{u}_{i}+\left(\mathbf{G}_{\psi i}^{e}-\frac{1}{\Omega^{e}} \int_{\Omega^{e}} \mathbf{G}_{\psi i}^{e} \mathrm{~d} V\right) \psi_{i}
$$

For interpolations of stress and electric flux density, the necessary (but not sufficient) conditions for element stability are:

$$
\begin{aligned}
& n_{\beta T} \geqslant n_{u}-m_{u} \\
& n_{\beta D} \geqslant n_{\phi}-m_{\phi}
\end{aligned}
$$

where $n_{\beta T}$ and $n_{\beta D}$ are the number of assumed stress and electric flux density modes, respectively. Furthermore, $n_{u}$ and $n_{\phi}$ are the number of displacement and potential modes, respectively. The number of rigid body displacement modes is $m_{u}$, and $m_{\phi}$ represents the single (constant potential) distribution resulting in zero field. The equality conditions in (42) and (43) represent the optimal number of parameters in both cases.

For our planar elements with drilling dof's, $n_{u}=12$, and $n_{\phi}=4$. The single potential distribution resulting in zero field means $m_{\phi}=1$, and usually membrane elements possess three 
rigid body modes $\left(m_{u}=3\right)$. The optimal number of parameters required is therefore $n_{\beta T}=9$ for stress and $n_{\beta D}=3$ for flux density. One more than optimal, i.e. $n_{\beta D}=4$, parameters are required for interpolation of the electric flux density in order to ensure the element is invariant [10]. Stability is achieved if no spurious zero energy modes appear, viz. if rank sufficiency of the mechanical stiffness and dielectric stiffness matrices is maintained [8].

Sze et al. [28,29] presented a rank sufficient elastic element with drilling dof's using only eight interpolating stress modes, which appears to be one less than the optimal prescribed in (42). However, $n_{\beta T}=8$ seems adequate, since $m_{u}$ in (42) is in fact equal to 4 and not 3 . The additional so-called $\theta_{1}$-mode (in which rotations at each node are equal) is intrinsic to the reduced displacement interpolation. The strain corresponding to this state is zero.

Geyer and Groenwold [33] also presented a family of membrane elements with drilling dof's, some of which employ only eight stress modes for interpolation and retain stability. We will test elements with both eight and nine stress interpolating modes.

The assumed electric flux density vector is interpolated using

$$
\mathbf{D}^{h}=\sum_{e}\left[\begin{array}{ll}
\mathbf{I}_{D c}^{e} & \mathbf{A}_{D}^{e} \mathbf{P}_{D h}^{e}
\end{array}\right]\left\{\begin{array}{l}
\boldsymbol{\beta}_{D c}^{e} \\
\boldsymbol{\beta}_{D h}^{e}
\end{array}\right\}
$$

where $\mathbf{I}_{D c}^{e}$ is a $2 \times 2$ identity matrix to account for the constant flux density case and $\boldsymbol{\beta}_{D c}^{e}$ are the corresponding parameters. $\mathbf{A}_{D}^{e}$ is a transformation matrix $[10,13,40]$, and $\mathbf{P}_{D h}^{e}$ represents the interpolation of the higher order part of charge density with $\boldsymbol{\beta}_{D h}^{e}$ the corresponding parameters. $\mathbf{A}_{D}^{e}$ and $\mathbf{P}_{D h}^{e}$ are given explicitly as

$$
\mathbf{A}_{D}^{e}=\left[\begin{array}{ll}
a_{1} & a_{3} \\
b_{1} & b_{3}
\end{array}\right]
$$

where for each element, $a_{i}$ and $b_{i}$ are based on the nodal co-ordinates $x$ and $y$ and are given by

$$
\left[\begin{array}{ll}
a_{1} & b_{1} \\
a_{2} & b_{2} \\
a_{3} & b_{3}
\end{array}\right]=\frac{1}{4}\left[\begin{array}{cccc}
-1 & 1 & 1 & -1 \\
1 & -1 & 1 & -1 \\
-1 & -1 & 1 & 1
\end{array}\right]\left[\begin{array}{ll}
x_{1} & y_{1} \\
x_{2} & y_{2} \\
x_{3} & y_{3} \\
x_{4} & y_{4}
\end{array}\right]
$$

The interpolation based on the local $\xi-\eta$ co-ordinates is given by

$$
\mathbf{P}_{D h}^{e}=\left[\begin{array}{ll}
\eta & 0 \\
0 & \xi
\end{array}\right]
$$

The interpolation for electric flux density $\mathbf{D}^{h}$ can therefore be given explicitly by

$$
\mathbf{D}^{h}=\sum_{e}\left[\begin{array}{cccc}
1 & 0 & a_{1} \eta & a_{3} \xi \\
0 & 1 & b_{1} \eta & b_{3} \xi
\end{array}\right]\left\{\begin{array}{l}
\boldsymbol{\beta}_{D c}^{e} \\
\boldsymbol{\beta}_{D h}^{e}
\end{array}\right\}
$$


The assumed symmetric part of stress is similarly interpolated as

$$
\operatorname{symm} \mathbf{T}^{h}=\sum_{e}\left[\begin{array}{ll}
\mathbf{I}_{T c}^{e} & \mathbf{A}_{T}^{e} \mathbf{P}_{T h n}^{e}
\end{array}\right]\left\{\begin{array}{l}
\boldsymbol{\beta}_{T c}^{e} \\
\boldsymbol{\beta}_{T h}^{e}
\end{array}\right\}
$$

where $\mathbf{I}_{T c}^{e}$ is a $3 \times 3$ identity matrix accounting for a constant stress state, with corresponding stress parameters $\boldsymbol{\beta}_{T c}^{e}$. $\mathbf{A}_{T}^{e}$ is a transformation matrix given by

$$
\mathbf{A}_{T}^{e}=\left[\begin{array}{ccc}
a_{1}^{2} & a_{3}^{2} & 2 a_{1} a_{3} \\
b_{1}^{2} & b_{3}^{2} & 2 b_{1} b_{3} \\
a_{1} b_{1} & a_{3} b_{3} & a_{1} b_{3}+a_{3} b_{1}
\end{array}\right]
$$

There are many other constraint matrices, (see for example Reference [41]), which can be used, but are not considered here for the sake of brevity. We implement two different higher order stress interpolations. The first has eight (i.e. five non-constant) $\boldsymbol{\beta}_{T}$-parameters and the other nine (six non-constant). The element with the eight $\boldsymbol{\beta}_{T}$-parameters has a higher order interpolation matrix given by

$$
\mathbf{P}_{T h 5}^{e}=\left[\begin{array}{ccccc}
\eta & 0 & -\xi & 0 & \eta^{2} \\
0 & \xi & 0 & -\eta & -\xi^{2} \\
0 & 0 & \eta & \xi & 0
\end{array}\right]
$$

which is similar to the field used by Sze and Ghali [29]. The element containing $9 \boldsymbol{\beta}_{T}$-parameters has a matrix given by

$$
\mathbf{P}_{T h 6}^{e}=\left[\begin{array}{cccccc}
\eta & 0 & -\xi & 0 & \eta^{2} & 0 \\
0 & \xi & 0 & -\eta & 0 & \xi^{2} \\
0 & 0 & \eta & \xi & 0 & 0
\end{array}\right]
$$

Alternative interpolations are given by Geyer and Groenwold [33].

\section{FINITE ELEMENT IMPLEMENTATION}

For simplicity, in this section we will neglect the boundary terms, which may be included in the usual manner. We also neglect the body charge $q$ terms, as is usually done [5]. In what is to follow, $\mathbf{u}^{h}$, symm $\mathbf{T}^{h}$, skew $\mathbf{T}^{h}, \phi^{h}, \mathbf{D}^{h}$ represent the interpolations for displacement, symmetric and skew-symmetric stress, electric potential and electric flux density, respectively. The fields $\mathbf{u}^{h}$ and $\phi^{h}$ satisfy the necessary boundary conditions, and $\psi^{h}$ denotes the interpolated rotation field.

Due to space considerations, only two elements derived from the foregoing variational formulations will be presented. The first is denoted P4dM and is derived from $\Pi_{\mathrm{M}}$. The second will be denoted $\mathrm{P} 4 \mathrm{dM} n \mathrm{TD}$ and is derived from $\Pi_{\mathrm{M}}^{T D}$. 


\subsection{P4dM element based on $\Pi_{\mathrm{M}}$}

The discrete form of the formulation based in $\Pi_{M}$ can be written in matrix form as

$$
\left[\begin{array}{ccc}
\mathbf{K}_{u u}^{e} & \mathbf{K}_{u \phi}^{e} & \mathbf{h}^{e} \\
{\left[\mathbf{K}_{u \phi}^{e}\right]^{\mathrm{t}}} & -\mathbf{K}_{\phi \phi}^{e} & \mathbf{0} \\
{\left[\mathbf{h}^{e}\right]^{\mathrm{t}}} & \mathbf{0} & -\gamma^{-1} \Omega^{e}
\end{array}\right]\left\{\begin{array}{c}
\mathbf{a} \\
\boldsymbol{\phi} \\
T_{0}^{e}
\end{array}\right\}=\left\{\begin{array}{l}
\mathbf{f} \\
\mathbf{0} \\
0
\end{array}\right\}, \quad \mathbf{a}=\left\{\begin{array}{c}
\mathbf{u} \\
\boldsymbol{\psi}
\end{array}\right\}
$$

The skew symmetric part of the stress tensor can be eliminated on the element level using static condensation, so that the system of equations necessary to solve the problem are

$$
\left[\mathbf{K}_{\mathrm{M}}^{e}\right]\left\{\begin{array}{l}
\mathbf{a} \\
\boldsymbol{\phi}
\end{array}\right\}=\left\{\begin{array}{l}
\mathbf{f} \\
\mathbf{0}
\end{array}\right\}
$$

where

$$
\mathbf{K}_{\mathrm{M}}^{e}=\left[\begin{array}{cc}
\mathbf{K}_{u u}^{e}+\frac{\gamma}{\Omega^{e}} \mathbf{h}^{e}\left[\mathbf{h}^{e}\right]^{\mathrm{t}} & \mathbf{K}_{u \phi}^{e} \\
{\left[\mathbf{K}_{u \phi}^{e}\right]^{\mathrm{t}}} & -\mathbf{K}_{\phi \phi}^{e}
\end{array}\right]
$$

Individual partitioned stiffness matrix terms are given in Appendix A.2. Both $\mathbf{K}_{u u}^{e}$ and $\mathbf{K}_{u \phi}^{e}$ are evaluated using a five-point numerical integration scheme, while $\mathbf{h}^{e}$ and $\mathbf{K}_{\phi \phi}^{e}$ employ a standard four-point scheme. The top left $(12 \times 12)$ portion of the stiffness matrix represents the mechanical part. The $12 \times 4$ partition, denoted $\mathbf{K}_{u \phi}^{e}$, represents the piezoelectric part, and the bottom right portion, $-\mathbf{K}_{\phi \phi}^{e}$, the $4 \times 4$ dielectric part of the stiffness matrix.

The skew part of the stress tensor (constant over each element) can be recovered as a post-processing step, and is given by

$$
T_{0}^{e}=\gamma \frac{\left[\mathbf{h}^{e}\right]^{\mathrm{t}} \mathbf{a}}{\Omega^{e}}
$$

5.2. P4dMnTD element based on $\Pi_{\mathrm{M}}^{T D}$

Considering functional $\Pi_{\mathrm{M}}^{T D}$, the discrete version of the formulation can be rewritten in matrix form as

$$
\left[\begin{array}{ccccc}
\mathbf{0} & \mathbf{0} & \mathbf{h}^{e} & \mathbf{K}_{u T}^{e} & \mathbf{0} \\
\mathbf{0} & \mathbf{0} & \mathbf{0} & \mathbf{0} & \mathbf{K}_{\phi D}^{e} \\
{\left[\mathbf{h}^{e}\right]^{\mathrm{t}}} & \mathbf{0} & -\gamma^{-1} \Omega^{e} & \mathbf{0} & \mathbf{0} \\
{\left[\mathbf{K}_{u T}^{e}\right]^{\mathrm{t}}} & \mathbf{0} & \mathbf{0} & -\mathbf{K}_{T T}^{T D e} & -\mathbf{K}_{T D}^{T D e} \\
\mathbf{0} & {\left[\mathbf{K}_{\phi D}^{e}\right]^{\mathrm{t}}} & \mathbf{0} & -\left[\mathbf{K}_{T D}^{T D e}\right]^{\mathrm{t}} & \mathbf{K}_{D D}^{T D e}
\end{array}\right]\left\{\begin{array}{c}
\mathbf{a} \\
\boldsymbol{\phi} \\
T_{0}^{e} \\
\boldsymbol{\beta}_{T}^{e} \\
\boldsymbol{\beta}_{D}^{e}
\end{array}\right\}=\left\{\begin{array}{l}
\mathbf{f} \\
\mathbf{0} \\
0 \\
\mathbf{0} \\
\mathbf{0}
\end{array}\right\}
$$


The matrix form of (57) can be simplified to

$$
\left[\mathbf{K}_{\mathrm{M}}^{T D e}\right]\left\{\begin{array}{l}
\mathbf{a} \\
\boldsymbol{\phi}
\end{array}\right\}=\left\{\begin{array}{l}
\mathbf{f} \\
\mathbf{0}
\end{array}\right\}
$$

where the stress and electric flux density $\beta$-parameters reduce to

$$
\left\{\begin{array}{l}
\boldsymbol{\beta}_{T}^{e} \\
\boldsymbol{\beta}_{D}^{e}
\end{array}\right\}=\left[\begin{array}{cc}
\mathbf{K}_{T T}^{T D e} & \mathbf{K}_{T D}^{T D e} \\
{\left[\mathbf{K}_{T D}^{T D e}\right]^{\mathrm{t}}} & -\mathbf{K}_{D D}^{T D e}
\end{array}\right]^{-1}\left[\begin{array}{cc}
{\left[\mathbf{K}_{u T}^{e}\right]^{\mathrm{t}}} & \mathbf{0} \\
\mathbf{0} & {\left[\mathbf{K}_{\phi D}^{e}\right]^{\mathrm{t}}}
\end{array}\right]\left\{\begin{array}{l}
\mathbf{a} \\
\boldsymbol{\phi}
\end{array}\right\}
$$

and the stiffness matrix for this element reduces to

$$
\mathbf{K}_{\mathrm{M}}^{T D e}=\left[\begin{array}{cc}
\frac{\gamma}{\Omega^{e}} \mathbf{h}^{e}\left[\mathbf{h}^{e}\right]^{\mathrm{t}} & \mathbf{0} \\
\mathbf{0} & \mathbf{0}
\end{array}\right]+\left[\begin{array}{cc}
\mathbf{K}_{u T}^{e} & \mathbf{0} \\
\mathbf{0} & \mathbf{K}_{\phi D}^{e}
\end{array}\right]\left[\begin{array}{cc}
\mathbf{K}_{T T}^{T D e} & \mathbf{K}_{T D}^{T D e} \\
{\left[\mathbf{K}_{T D}^{T D e}\right]^{\mathrm{t}}} & -\mathbf{K}_{D D}^{T D e}
\end{array}\right]^{-1}\left[\begin{array}{cc}
{\left[\mathbf{K}_{u T}^{e}\right]^{\mathrm{t}}} & \mathbf{0} \\
\mathbf{0} & {\left[\mathbf{K}_{\phi D}^{e}\right]^{\mathrm{t}}}
\end{array}\right]
$$

Again, individual partitioned stiffness matrix terms are given in Appendix A.2. The partitioned submatrices $\mathbf{K}_{u T}^{e}, \mathbf{K}_{T T}^{T D e}$ and $\mathbf{K}_{T D}^{T D e}$ are calculated using a five-point integration scheme, while $\mathbf{K}_{\phi D}^{e}$ and $\mathbf{K}_{D D}^{T D e}$ are evaluated using a four-point scheme. The number of stress parameters in $\boldsymbol{\beta}_{T}^{e}$ is denoted $n$ in the element designation P4dM $n$ TD.

\section{NUMERICAL EVALUATION}

In this section, the developed piezoelectric finite elements are assessed numerically and compared with existing elements. The effect of selected parameters are also quantified. For brevity, only the two elements presented in Section 5 are reported on. However, eight different elements resulting from the formulations herein have been implemented. Further information regarding the performance of the other elements is available from the first author on request [37]. The elements used in the comparison are denoted as follows:

- P4-a standard, planar four node, quadrilateral piezoelectric element without drilling dof's. Only the displacement $\mathbf{u}$ and the potential $\phi$ are interpolated for, see for example Reference [3].

- P4TD-a mixed four node element with both stress $\mathbf{T}$ and electric flux density $\mathbf{D}$ assumed additionally to displacement and electric potential, as proposed in Reference [10].

- P4dM- a four node element with drilling dof's, derived from functional $\Pi_{M}$ in (27), and with stiffness matrix given by (55).

- P4dMnTD—a mixed four node element, based on functional $\Pi_{\mathrm{M}}^{T D}$ given in (29), with interpolations for $\mathbf{u}, \phi, \mathbf{T}$ and $\mathbf{D}$. The number of stress interpolation parameters is given by $n$. The stiffness matrix of $\mathrm{P} 4 \mathrm{dM} n \mathrm{TD}$ is given in (60).

In the test problems to follow, unless otherwise stated, the material constants of PZT-4 given by Sze et al. [9] are employed so that our piezoelectric elements may be compared to theirs. 
To this end, the following material constants are used:

$$
\begin{aligned}
& c_{11}=139 \times 10^{3}, \quad c_{33}=113 \times 10^{3}, \quad c_{13}=74.3 \times 10^{3}, \quad c_{55}=25.6 \times 10^{3} \quad\left(\text { in } \mathrm{N} / \mathrm{mm}^{2}\right) \\
& c_{15}=13.44 \times 10^{6}, \quad e_{31}=-6.98 \times 10^{6}, \quad e_{33}=13.84 \times 10^{6}\left(\text { in } \mathrm{pC} / \mathrm{mm}^{2}\right) \\
& \varepsilon_{11}=6.00 \times 10^{9}, \quad \varepsilon_{33}=5.47 \times 10^{9} \quad(\text { in } \mathrm{pC} /(\mathrm{GVmm})) .
\end{aligned}
$$

The units of length, force, stress, charge, electric displacement and electric potential, respectively, are taken as $\mathrm{mm}, \mathrm{N}, \mathrm{N} / \mathrm{mm}^{2}, \mathrm{pC}, \mathrm{pC} / \mathrm{mm}^{2}$ and $\mathrm{GV}$. This unusual unit choice alleviates the ill effects resulting from the poor scaling of the global stiffness matrix. The poling direction in the test problems to follow, unless otherwise stated, is taken as the global $y$-direction. The constitutive equations which result are

$$
\left\{\begin{array}{c}
T_{x x} \\
T_{y y} \\
T_{x y} \\
D_{x} \\
D_{y}
\end{array}\right\}=\left[\begin{array}{ccccc}
c_{11} & c_{13} & 0 & 0 & -e_{31} \\
c_{13} & c_{33} & 0 & 0 & -e_{33} \\
0 & 0 & c_{55} & -e_{15} & 0 \\
0 & 0 & e_{15} & \varepsilon_{11} & 0 \\
e_{31} & e_{33} & 0 & 0 & \varepsilon_{33}
\end{array}\right]\left\{\begin{array}{c}
S_{x x} \\
S_{y y} \\
S_{x y} \\
E_{x} \\
E_{y}
\end{array}\right\}
$$

Constants used in the analytical solutions of some of the tests to follow, are given by

$$
\left[\begin{array}{ccc}
s_{11} & s_{13} & g_{31} \\
s_{13} & s_{33} & g_{33} \\
-g_{31} & -g_{33} & \beta_{33}
\end{array}\right]=\left[\begin{array}{ccc}
c_{11} & c_{13} & -e_{31} \\
c_{13} & c_{33} & -e_{33} \\
e_{31} & e_{33} & \varepsilon_{33}
\end{array}\right]^{-1}
$$

\subsection{Effect of $\gamma$}

The elements developed in the foregoing are dependant on the problem-dependent penalty parameter $\gamma$. The effect of $\gamma$ has been the focus of a number of recent studies [33, 42, 43]. For linear elastic isotropic Dirichlet problems, the formulation is reported to be relatively insensitive to the value of $\gamma[23,26,44]$, and it was proposed that $\gamma=\mu$, the shear modulus. Under different conditions, e.g. orthotropy (as in the case of piezoelectric materials) or dynamic problems, a greater sensitivity to $\gamma$ is expected. For dynamic problems, for example, Hughes et al. [45] propose a value of $\gamma=\mu / 10$.

To determine an appropriate value for $\gamma$, an eigenvalue analysis of an undistorted (square) $\mathrm{P} 4 \mathrm{dM}$ element is performed. Although results are only reported for the P4dM element, the trends depicted in Figures 2 and 4 are typical for all of our new elements. Figure 2 depicts the effect of $\gamma$ on the non-zero eigenvalues, arranged in descending order. The eigenvalues are normalized with respect to their values at $\gamma=c_{33}$. It is clear that the 'softer' modes are most sensitive to $\gamma$ at values of $\gamma / c_{33}<1$. For values of $\gamma / c_{33}>1$, on the other hand, the 'harder' modes are significantly more sensitive, in particular $\lambda_{1}$ and $\lambda_{2}$, indicative of a locking-like phenomena. This results from the terms in the stiffness matrix containing $\gamma$ dominating the response. 


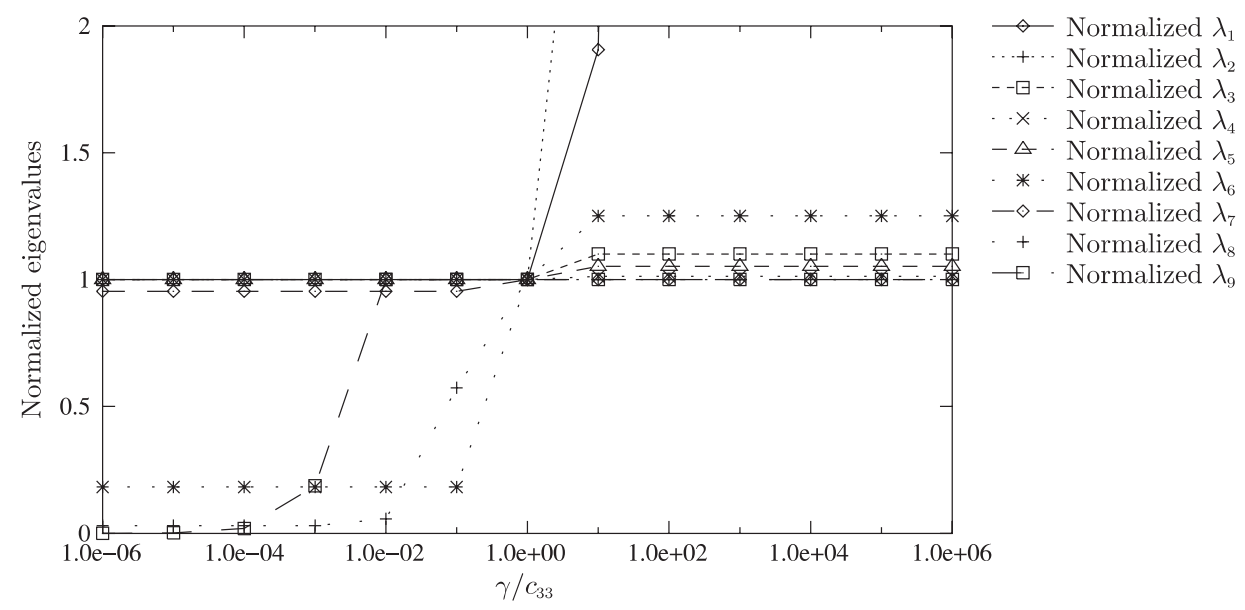

Figure 2. Effect of $\gamma$ on eigenvalues (normalized with respect to their values at $\gamma / c_{33}=1$ ).

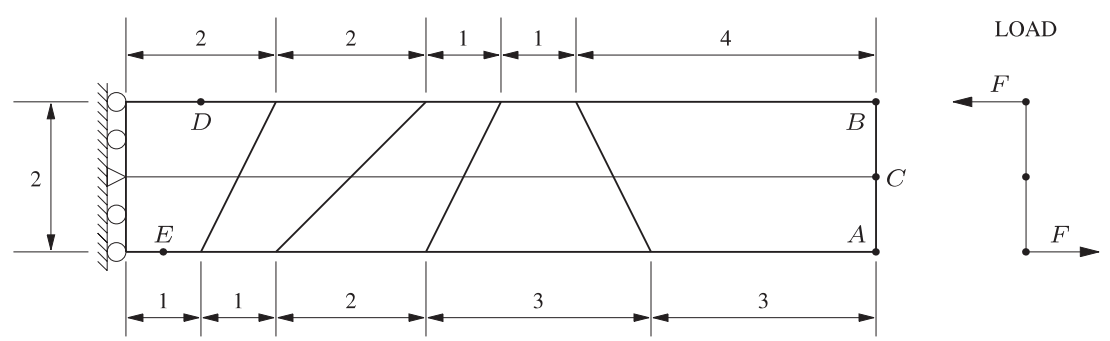

Figure 3. Ten element cantilever beam subjected to pure bending.

In order to determine the effect of $\gamma$ on the accuracy of the current formulations, a representative test problem is studied for an array of $\gamma$ values. The 10 element cantilever, depicted Figure 3, is selected for this study. In Figure 4 some accuracy measures are plotted as a function of $\gamma / c_{33}$.

Figure 4 shows how the accuracy of $u_{y B}$, the $y$-displacement at point $B$ and of $\phi_{C}$, the electric potential at $C$ (see Figure 3), decrease at values of $\gamma / c_{33}>1$. Also plotted is an indication of the error on the skew-symmetric part of stress, $T_{0}$. The plotted values are normalized with respect to principle stresses at points $D$ and $E$. $T_{0 D}$ and $T_{0 E}$ are the constant skew-symmetric part of stress in the elements containing points $D$ and $E$, respectively. $T_{2 D}$ is the second (compressive) principle stress calculated at $D$ and $T_{1 E}$ is the first principle stress at $E$. Since the skew-symmetric part of stress should be zero, both $T_{0 D} / T_{2 D}$ and $T_{0 E} / T_{1 E}$ should in turn be zero. Notably, the error on the skew-symmetric stress increases with larger values of $\gamma / c_{33}$. To avoid operating in the regime where the gradient change of the accuracy measures occurs, we suggest the use of $\gamma / c_{33}=10^{-2}$. 


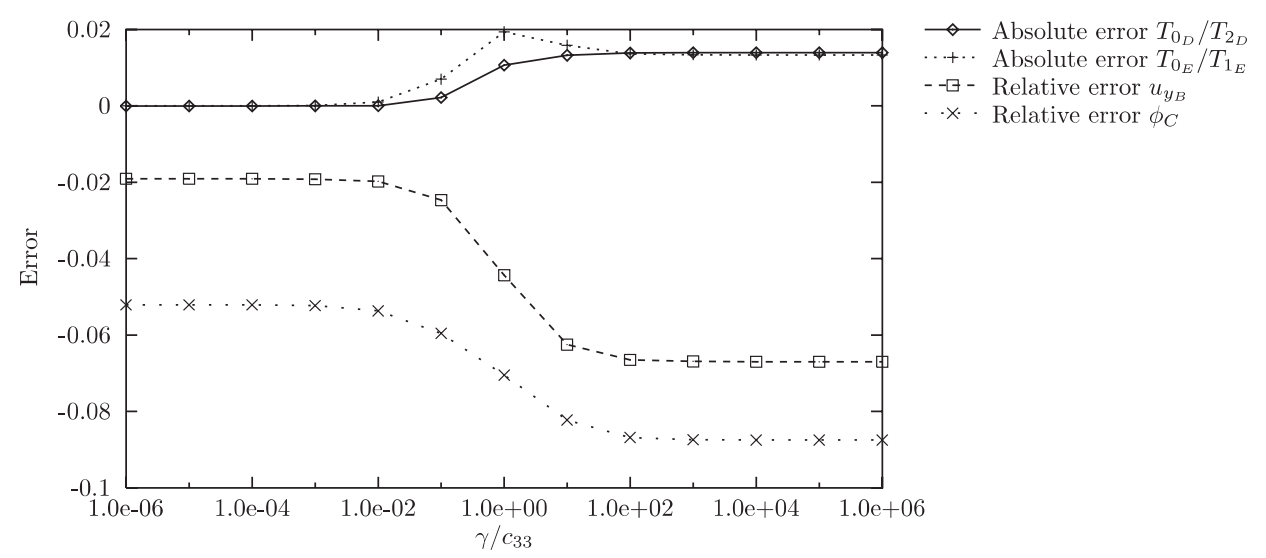

Figure 4. Effect of $\gamma$ on skew part of stress and other accuracy measures.

\subsection{Eigenvalue analysis}

Since a reduced integration scheme is employed during stiffness calculations and a nonstandard $\gamma$ value is used, element rank sufficiency needs to be verified by means of an eigenvalue analysis. The eigenvalues of $2 \times 2$, undistorted (square) elements are computed. (Eigenvalue analyses should in general be carried out on undistorted elements, since distortion may actually suppress zero energy modes due to the inaccuracies of an approximate integration scheme.)

In the case of planar piezoelectric elements, the mechanical partition of the stiffness matrix should contain only three zero eigenvalues corresponding to the three rigid body modes. An eigenvalue analysis of the dielectric part of the element stiffness matrix should reveal a single zero eigenvalue, corresponding to the constant potential distribution resulting in zero electric field. For the sake of brevity, the eigenvalues are not reported here. It was, however verified that each element possesses the proper number of non-zero eigenvalues.

\subsection{Patch test}

The patch test is a standard method to test for element convergence, as well as any possible implementation or programming errors. We perform the test with the geometry and mesh suggested by Sze et al. [9], as shown in Figure 5. Kinematic (displacement and potential) terms on the boundary are prescribed, corresponding to

$$
u_{x}=s_{11} \sigma_{0} x, \quad u_{y}=s_{13} \sigma_{0} y, \quad \phi=g_{31} \sigma_{0} y
$$

with $\sigma_{0}$ a stress parameter. The corresponding stress and electric displacement can be shown to be constant, and are given by

$$
T_{x x}=\sigma_{0}, \quad T_{y y}=T_{x y}=D_{x}=D_{y}=0
$$

Compliance with the conditions above was verified for each of the elements used in this study. A force patch test, with prescribed boundary forces corresponding to (64), was also conducted. 


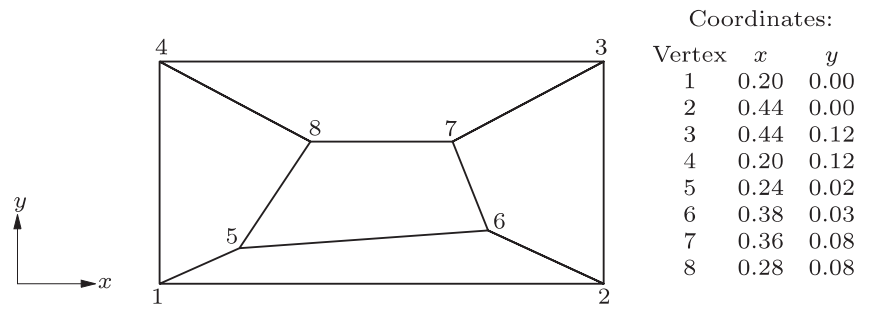

Figure 5. Mesh for piezoelectric patch test.

\subsection{Two element beam}

The two element beam bending test is used to quantify the effect of distortion on element accuracy. Figure 6 depicts a cantilever beam of length $L=10$ and height $h=2$, modelled using two elements with a common distortion $e$. The electric potential of all nodes at $y=-1$ is prescribed to be zero. The exact solution for this problem was presented by Sze et al. [9]. The analytical solutions to this problem include:

$$
\begin{aligned}
& u_{x}=-s_{11} \sigma_{0} x y, \quad u_{y}=\frac{s_{13} \sigma_{0}}{2}\left(\frac{h^{2}}{4}-y^{2}\right)+\frac{s_{11}}{2} \sigma_{0} x^{2} \\
& \phi=\frac{g_{31} \sigma_{0}}{2}\left(\frac{h^{2}}{4}-y^{2}\right), \quad T_{x}=-\sigma_{0} y \\
& T_{y}=T_{x y}=D_{x}=D_{y}=0, \quad M=\int_{-h / 2}^{h / 2} y T_{x} \mathrm{~d} y=-\frac{h^{3} \sigma_{0}}{12}=-h F
\end{aligned}
$$

Figure 7 depicts the relative error of the $y$-displacement at point $A$, i.e. $u_{y_{A}} / u_{y \text { Exact }}-1$, for the various elements being tested. Considering the two irreducible elements, the superior accuracy and stability of the P4dM element over the standard P4 elements is clear. It is also demonstrated that the P4dM8TD element is accurate and stable, even at extreme mesh distortions. In fact, for this problem P4d8MTD performs slightly better than the stabilized plane element developed by Sze et al. [9], as shown in Figure 7.

Figure 8 depicts the absolute error on the electric potential at point $A, \phi_{A}$. The two irreducible elements, P4 and P4dM, achieve the best potential approximations. However, the parabolic 'through-thickness' potential distribution can, of course, not be captured using bilinear potential interpolations with the current mesh. The result is that for a regular mesh, zero electric field is predicted and therefore relatively large errors on displacement accrue due to the inherent coupling. In this instance, our two new elements are more sensitive to mesh distortion than the P4TD element. However, if a similar test is conducted with two elements through the thickness, i.e. four elements in total, the predictions of the mixed elements are similar. (Results for this mesh are not explicitly shown here.) This, in our opinion, is a more reasonable mesh for this problem, since the physics of the problem can be approximated by all elements used in the comparison. That is to say, the parabolic potential distribution can be approximated using the bilinear potential interpolations of the aforementioned elements. Unfortunately Sze et al. [9] did not report on this quantity, so comparison with their element was not possible. 


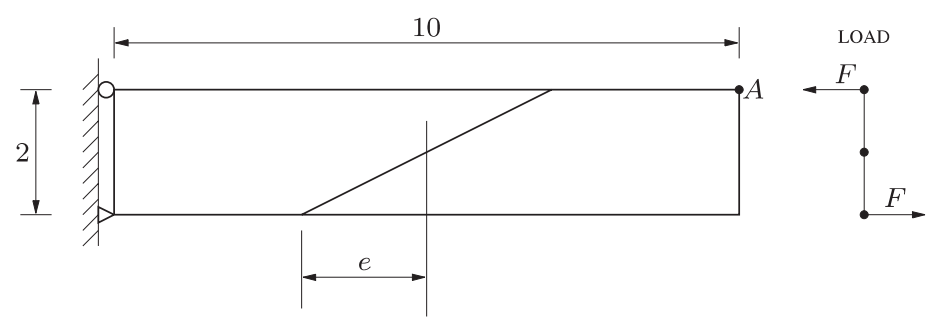

Figure 6. Two element cantilever beam subject to pure bending.

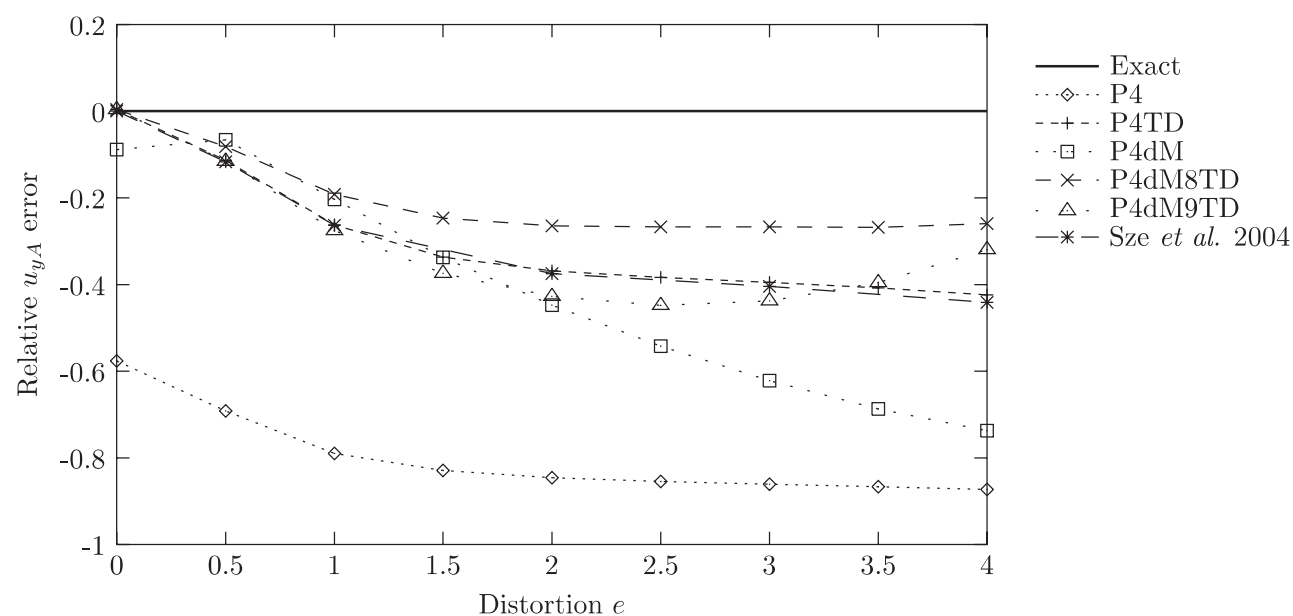

Figure 7. Two element beam: effect of distortion on $u_{y A}$.

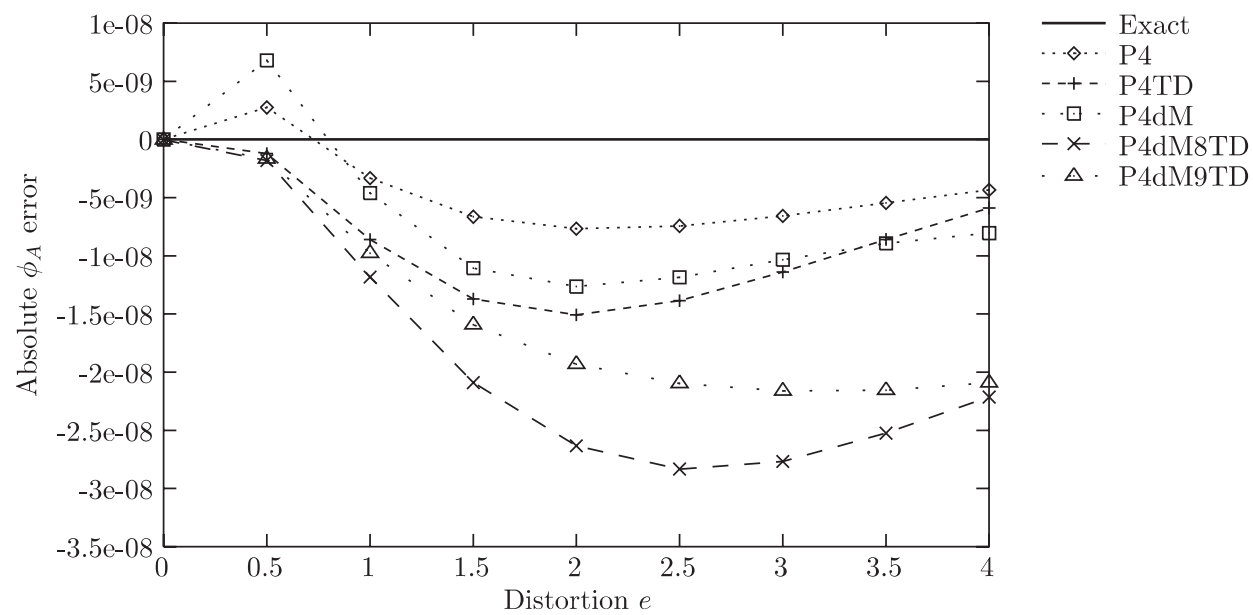

Figure 8. Two element beam: effect of distortion on $\phi_{A}$. 
Table I. Ten element cantilever subject to pure bending.

\begin{tabular}{|c|c|c|c|c|c|c|c|}
\hline & $\begin{array}{c}\text { Relative } \\
\% \text { error } \\
u_{x B}\end{array}$ & $\begin{array}{c}\text { Relative } \\
\% \text { error } \\
u_{y B}\end{array}$ & $\begin{array}{c}\text { Relative } \\
\% \text { error } \\
\phi_{C}\end{array}$ & $\begin{array}{c}\text { Relative } \\
\% \text { error } \\
T_{x D}\end{array}$ & $\begin{array}{c}\text { Relative } \\
\% \text { error } \\
T_{x E}\end{array}$ & $\begin{array}{c}\text { Absolute } \\
\text { error } \\
D_{y D}\end{array}$ & $\begin{array}{c}\text { Absolute } \\
\text { error } \\
D_{y E}\end{array}$ \\
\hline 24 & -40.4175 & -36.1072 & -48.1191 & -21.3819 & -5.2331 & 90.1438 & -10.0964 \\
\hline P4TD & -5.7885 & -4.0462 & 1.7121 & 0.7077 & 0.4331 & -6.6586 & 7.4355 \\
\hline P4dM & -3.4094 & -1.3680 & -5.3626 & 9.2982 & 18.1271 & 97.7308 & -102.4166 \\
\hline P4d & -6.4972 & -4.8806 & & & & & 8.3963 \\
\hline P4dM9TD & -6.4364 & -4.8969 & 1.0597 & -0.9267 & 7.1655 & -4.3515 & 8.4282 \\
\hline
\end{tabular}

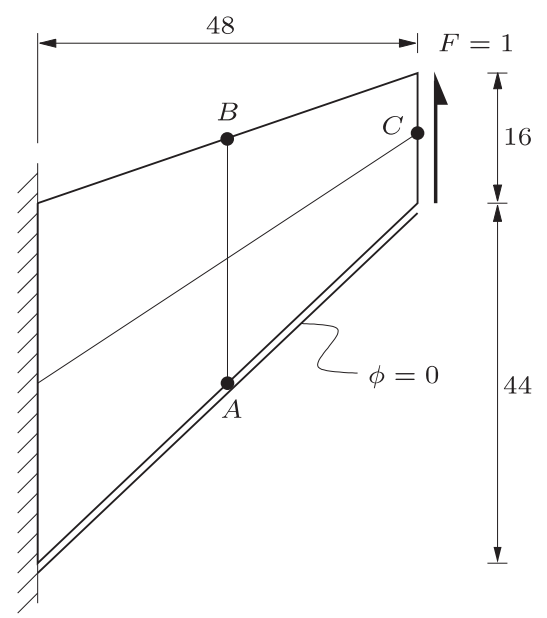

Figure 9. Cook's membrane.

\subsection{Ten element beam}

The same beam geometry as used in the two element test (with identical boundary conditions) is modelled, but this time with 10 irregular elements as shown in Figure 3. Displacements and electric potentials are evaluated at points $A, B$ and $C$ and stresses and electric flux densities are calculated directly at points $D$ and $E$. This problem was used by Wu et al. [10] to verify the accuracy of their P4TD element. Results are presented in Table I. Again, considering the two irreducible elements used in this comparison, the superior accuracy with respect most fields of the P4dM element relative to P4 is evident. For this specific problem, all of the elements with stress and electric flux density assumed, exhibit a similar good performance.

\subsection{Cook's membrane}

The final pathological test of element accuracy under mechanical actuation is depicted in Figure 9. The geometry and loading is similar to the popular Cook's membrane consisting of a swept and tapered beam with distributed tip load. The lower surface is prescribed to have a voltage of $0 \mathrm{~V}$. Since no analytical solution exists for this problem, the predicted solutions 


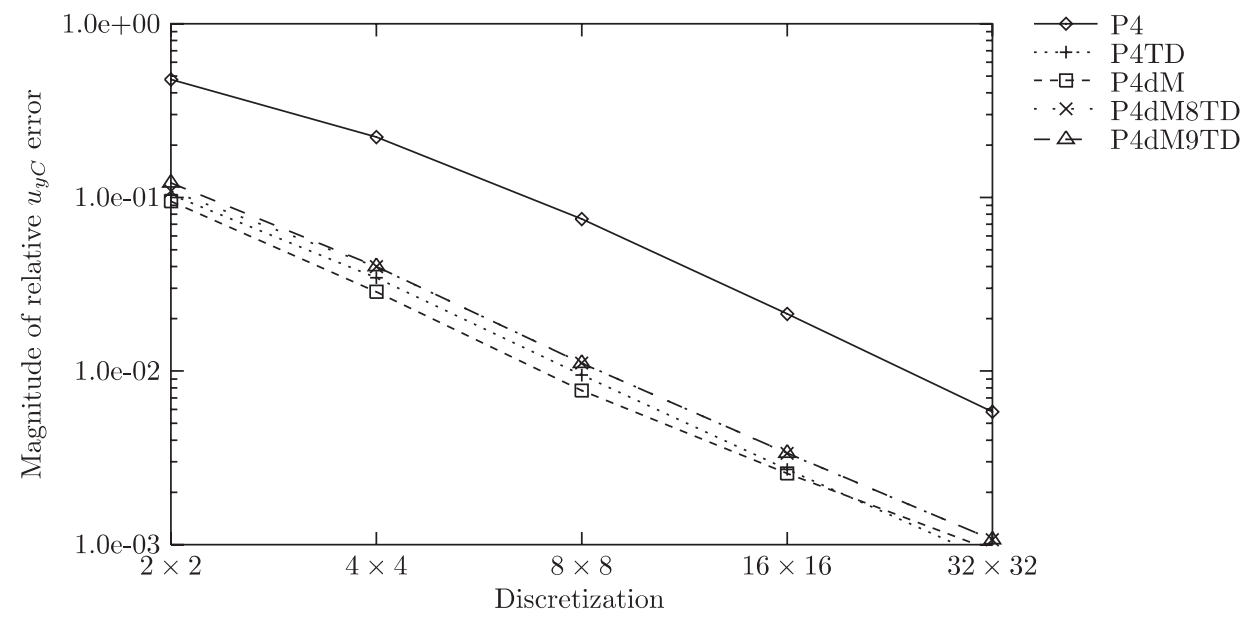

Figure 10. Cook's membrane: $y$-displacement at $C\left(u_{y C}\right)$.
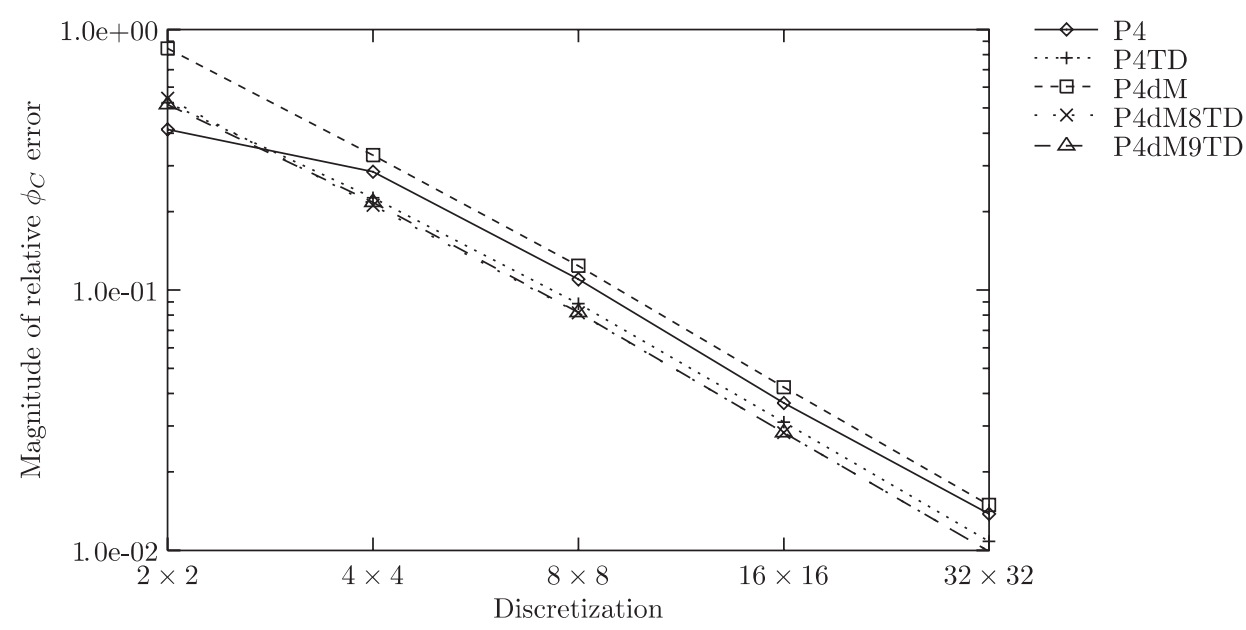

Figure 11. Cook's membrane: electric potential at $C\left(\phi_{y C}\right)$.

are compared to a finite element approximation with a fine mesh. The best known values of $u_{y C}$ and $\phi_{C}$ are $2.109 \times 10^{-4} \mathrm{~mm}$ and $1.732 \times 10^{-8} \mathrm{GV}$, respectively. Figure 10 depicts the magnitude of the relative error on $u_{y C}$, the $y$-displacement of point $C$, for different mesh refinements on a $\log$ scale. The relative accuracy of the P4dM element is again illustrated, and in fact, $\mathrm{P} 4 \mathrm{dM}$ achieves the best prediction for $u_{y c}$. On the whole, all the mixed elements perform similarly well.

Figure 11 depicts the magnitude of the relative error on $\phi_{C}$, the electric potential at point $C$. The two new mixed elements, P4dM8TD and P4dM9TD, perform slightly better than the $\mathrm{P} 4 \mathrm{TD}$ element in this case. Once again $\mathrm{P} 4 \mathrm{dM}$ is superior to $\mathrm{P} 4$. 
Table II. Relative percentage error for stress and electric displacement for Cook's membrane.

\begin{tabular}{|c|c|c|c|c|c|c|}
\hline & \multicolumn{2}{|c|}{$2 \times 2 \mathrm{mesh}$} & \multicolumn{2}{|c|}{$4 \times 4$ mesh } & \multicolumn{2}{|c|}{$8 \times 8$ mesh } \\
\hline & $T_{1 A}$ & $\left|D_{B}\right|$ & $T_{1 A}$ & $\left|D_{B}\right|$ & $T_{1 A}$ & $\left|D_{B}\right|$ \\
\hline P4 & -44.6706 & -80.3136 & -19.8108 & -68.3021 & -3.7775 & -33.9002 \\
\hline P4TD & -20.9579 & -45.4065 & -6.0842 & -23.5878 & -1.6060 & -10.6112 \\
\hline P4dM & -9.2049 & -38.4155 & -0.7867 & -9.7136 & -0.7298 & -6.7096 \\
\hline P4dM8TD & -35.3104 & -48.9795 & -7.3783 & -25.3382 & -2.1842 & -11.6869 \\
\hline P4dM9TD & -18.2960 & -47.2924 & -4.7375 & -25.4509 & -1.3131 & -11.6292 \\
\hline
\end{tabular}

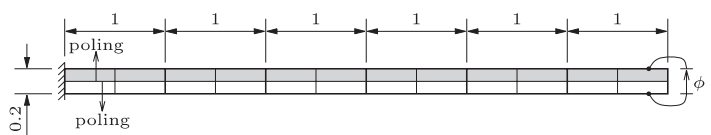

(a)

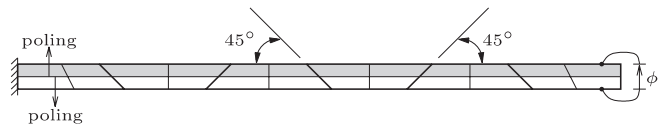

(b)

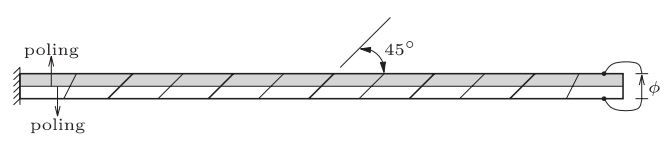

(c)

Figure 12. Bimorph based on MacNeal's elongated beam: (a) regular shape elements; (b) trapezoidal shape elements; and (c) parallelogram shape elements.

Table II presents the relative percentage errors on the first principle stress at point $A, T_{1 A}$ and the magnitude of the electric flux density at $B,\left|D_{B}\right|$. Again, the P4dM performs very well compared to the P4 element. Once again, all the four field elements perform similarly well. In this case P4dM9TD performs slightly better than the other four field elements on stress predictions, while P4TD achieves the greatest accuracy on electric flux density. The best known values for $T_{1 A}$ and $\left|D_{B}\right|$ are $0.21613 \mathrm{~N} / \mathrm{mm}^{2}$ and $22.409 \mathrm{pC} / \mathrm{mm}^{2}$, respectively.

\subsection{Piezoelectric bimorph beam}

The final problem, evaluating element accuracy under electrical actuation, takes the form of a piezoelectric bimorph beam. The physical problem consists of two identical layers of piezoelectric material with opposite polarities, as indicated in Figure 12. Upon application of an electric field in the through-thickness direction, the bimorph bends as a result of moments caused by the layers' opposing polarities.

This problem is often solved using PVDF material constants and compared to the solution of Tzou [46]. For the purpose of the current study, tests are conducted using two different materials. To assess the element accuracy by beam solution, the Poisson's ratio is set to zero. Therefore, the material properties for the PVDF material are [47] $E_{1}=E_{2}=E_{3}=2 \times 10^{3}\left(\mathrm{~N} / \mathrm{mm}^{2}\right)$, $v_{12}=v_{13}=v_{23}=0, \quad e_{31}=e_{32}=-0.046 \times 10^{6} \quad\left(\mathrm{pC} / \mathrm{mm}^{2}\right) \quad$ and $\quad \varepsilon_{11}=\varepsilon_{33}=\varepsilon_{33}=0.1062 \times 10^{9}$ $(\mathrm{pC} / \mathrm{GVmm})$. The $e_{33}$ coefficient is assumed to be zero [47]. In the second case, the material properties of PZT-4, as used in the preceding problems, are used and the solution is compared to a refined finite element solution. 
Table III. Relative percentage error on vertical tip displacement of piezoelectric bimorph.

\begin{tabular}{|c|c|c|c|c|c|c|}
\hline & \multicolumn{3}{|c|}{ PVDF } & \multicolumn{3}{|c|}{ PZT-4 } \\
\hline & Regular & Trapezoidal & Parallel & Regular & Trapezoidal & Parallel \\
\hline P4 & -75.7576 & -85.7547 & -88.7211 & -63.2273 & -80.9720 & -87.5199 \\
\hline P4TD & -0.2484 & -29.3670 & -4.4527 & -2.6514 & -26.4455 & -5.2251 \\
\hline P4dM & 0.0 & -35.4700 & -10.4663 & -6.3609 & -27.7086 & 1.9377 \\
\hline P4dM8TD & -0.2484 & -33.9872 & -5.2288 & -2.9145 & -29.0260 & -5.5317 \\
\hline P4dM9TD & -0.2484 & -34.0154 & -5.2717 & -3.2111 & -29.4803 & -5.8180 \\
\hline
\end{tabular}

Since at least two elements are required through the thickness, the meshes used here represents a bisection of the discretizations suggested by MacNeal and Harder [48]. The top surface of the beam is subjected to $1 \mathrm{~V}$, and the bottom surface to $-1 \mathrm{~V}$. The relative percentage error on the tip displacement is reported for the two different materials and the three meshes in Table III. The beam solution for the PVDF material is $6.2100 \times 10^{-5} \mathrm{~mm}$, while the best known solution for the PZT-4 material is $4.3622 \times 10^{-4} \mathrm{~mm}$.

The poor performance of the P4 element is once again highlighted. For this problem, the displacement predictions for the distorted meshes of P4TD are slightly better than the P4dM8TD and P4dM9TD elements. The irreducible element with drilling dof's, P4dM, demonstrates a performance comparable with the mixed elements.

\section{CLOSURE}

We have presented a number of variational formulations accounting for piezoelectricity and in-plane rotations. We have introduced two new families of functionals, namely M-Type, which retains the skew-symmetric part of the stress tensor, and K-Type, in which the skew part of stress is eliminated.

From the M-Type Hu-Washizu-like functional we have developed an irreducible formulation with only 'kinematic' independent variables, i.e. displacement and electric potential. We have also shown how a 'fully' mixed formulation, with stress and electric flux density assumed, can be generated. Two M-Type 'degenerate' Hellinger-Reissner-like functionals with either stress or electric flux density assumed, are also given. Due to space considerations, their formulations are, however, not presented. We have further indicated how the K-Type counterparts of our M-Type functionals can be constructed.

For the sake of brevity, only numerical implementations of selected M-Type finite elements are presented. We have demonstrated the accuracy and robustness of our elements on a number of benchmark problems. The addition of drilling dof's enriches the interpolated displacement field, resulting in improved element performance. This is borne out by the improved accuracy and robustness of the P4dM element over the standard P4 piezoelectric element. The improved performance of our mixed elements with drilling dof's is less marked when compared to existing mixed piezoelectric elements. In fact, it is difficult to conclusively state that any one of the elements used in this study is better in terms of accuracy than all the others. This is so since none of the elements herein consistently outperforms all the other elements on all reported accuracy measures. Our 'fully mixed' elements, however, are shown to be accurate and stable, 
even at extreme element distortions. They also allow for improved modelling capabilities due to the additional rotational dof, e.g. compatibility with elastic elements with drilling dof's is ensured. The results presented herein, therefore indicate that the $\mathrm{P} 4 \mathrm{dM} n \mathrm{TD}$ elements are useful for modelling engineering applications.

What is more, the variational formulations constructed in this work can be used to establish three-dimensional solid piezoelectric elements with drilling dof's. Furthermore, the planar elements derived here, when combined with piezoelectric plate elements, can be used to calculate through-thickness phenomena in thick piezoelectric shell elements.

\section{APPENDIX A}

\section{A.1. Element operators}

In (39), the operators $\mathbf{B}_{i}$ and $\mathbf{G}_{\psi i}$ are given as

$$
\mathbf{B}_{i}=\left[\begin{array}{cc}
N_{i, 1} & 0 \\
0 & N_{i, 2} \\
N_{i, 2} & N_{i, 1}
\end{array}\right], \quad i=1,2,3,4
$$

with $N_{i}, i=1,2,3,4$, the Lagrangian interpolation functions. The strain operator associated with the drilling rotation is defined by

$$
\left.\mathbf{G}_{\psi i}=\frac{1}{8}\left[\begin{array}{c}
\left(l_{i j} \cos \alpha_{i j} N_{l, 1}-l_{i k} \cos \alpha_{i k} N_{m, 1}\right) \\
\left(l_{i j} \sin \alpha_{i j} N_{l, 2}-l_{i k} \sin \alpha_{i k} N_{m, 2}\right) \\
l_{i j} \cos \alpha_{i j} N_{l, 2}-l_{i k} \cos \alpha_{i k} N_{m, 2} \\
+ \\
l_{i j} \sin \alpha_{i j} N_{l, 1}-l_{i k} \sin \alpha_{i k} N_{m, 1}
\end{array}\right\}\right]
$$

where $l_{j k}$ represent the lengths of sides $j k$ and, using a FORTRAN pseudo-language,

$$
\begin{aligned}
i & =1,2,3,4 ; \quad m=i+4 ; \quad l=m-1+4 \operatorname{int}(1 / i) \\
k & =\bmod (m, 4)+1 ; \quad j=l-4
\end{aligned}
$$

The functions $N_{i}, i=5,6,7,8$ are serendipity mid-side interpolation functions.

The operators associated with the skew-symmetric part of the displacement gradient are

$$
\mathbf{b}_{i}=\left\langle-\frac{1}{2} N_{i, 2} \frac{1}{2} N_{i, 1}\right\rangle, \quad i=1,2,3,4
$$

and

$$
\begin{aligned}
g_{i}= & -\frac{1}{16}\left(l_{i j} \cos \alpha_{i j} N_{l, 2} l_{i k} \cos \alpha_{i k} N_{m, 2}\right) \\
& +\frac{1}{16}\left(l_{i j} \sin \alpha_{i j} N_{l, 1} l_{i k} \sin \alpha_{i k} N_{m, 1}\right)-N_{i}, \quad i=1,2,3,4
\end{aligned}
$$


with indices $j, k, l, m$ again defined by (A3). In (35), a FORTRAN-like definition of adjacent corner nodes is also employed:

$$
j=i-4, \quad k=\bmod (i, 4)+1
$$

\section{A.2. Partitioned stiffness matrices}

The partitioned stiffness matrices are given in matrix form by

$$
\begin{aligned}
& \mathbf{K}_{u u}^{e}{ }^{(5)}=\int_{\Omega^{e}}\left[\begin{array}{ll}
\mathbf{B}_{u}^{e} & \mathbf{G}_{u}^{e}
\end{array}\right]^{\mathrm{t}} \mathbf{c}_{E}\left[\begin{array}{ll}
\mathbf{B}_{u}^{e} & \mathbf{G}_{u}^{e}
\end{array}\right] \mathrm{d} V \\
& \mathbf{K}_{u \phi}^{e}{ }^{(5)}=\int_{\Omega^{e}}\left[\mathbf{B}_{u}^{e} \mathbf{G}_{u}^{e}\right]^{\mathrm{t}} \mathbf{e}^{\mathrm{t}}\left[\mathbf{B}_{\phi}^{e}\right] \mathrm{d} V \\
& \mathbf{K}_{\phi \phi}^{e}{ }^{(4)}=\int_{\Omega^{e}}\left[\mathbf{B}_{\phi}^{e}\right]^{\mathrm{t}} \boldsymbol{\varepsilon}_{S}\left[\mathbf{B}_{\phi}^{e}\right] \mathrm{d} V \\
& \mathbf{h}^{e(4)}=\int_{\Omega^{e}}\left[\begin{array}{ll}
\mathbf{b}^{e} & \mathbf{g}^{e}
\end{array}\right]^{\mathrm{t}} \mathrm{d} V \\
& \mathbf{K}_{\phi D}^{e}{ }^{(4)}=\int_{\Omega^{e}}\left[\mathbf{B}_{\phi}^{e}\right]^{t}\left[\mathbf{P}_{D}^{e}\right] \mathrm{d} V \\
& \mathbf{K}_{u T}^{e}{ }^{(5)}=\int_{\Omega^{e}}\left[\begin{array}{ll}
\mathbf{B}_{u}^{e} & \mathbf{G}_{u}^{e}
\end{array}\right]^{\mathrm{t}}\left[\mathbf{P}_{T}^{e}\right] \mathrm{d} V \\
& \mathbf{K}_{T D}^{T D e(5)}=\int_{\Omega^{e}}\left[\mathbf{P}_{T}^{e}\right]^{\mathrm{t}} \mathbf{g}^{\mathrm{t}}\left[\mathbf{P}_{D}^{e}\right] \mathrm{d} V \\
& \mathbf{K}_{T T}^{T D e(5)}=\int_{\Omega^{e}}\left[\mathbf{P}_{T}^{e}\right]^{\mathrm{t}} \mathbf{s}_{D}\left[\mathbf{P}_{T}^{e}\right] \mathrm{d} V \\
& \mathbf{K}_{D D}^{T D e(4)}=\int_{\Omega^{e}}\left[\mathbf{P}_{D}^{e}\right]^{\mathrm{t}} \chi_{T}\left[\mathbf{P}_{D}^{e}\right] \mathrm{d} V
\end{aligned}
$$

The superscript in parentheses $(\cdot)$ represents the number of integration points used to perform the numerical integration.

\section{ACKNOWLEDGEMENTS}

This research was performed as part of the South African NRF THRIP Project 2769-Development of Smart Micropositioning Technology. Their financial support is gratefully acknowledged.

\section{REFERENCES}

1. Heyliger P. A note on the static behaviour of simply-supported laminated piezoelectric cylinders. International Journal of Solids and Structures 1997; 34:3781-3794. 
2. Kapuria S, Sengupta S, Dumir PC. Three-dimensional solution for simply-supported piezoelectric cylindrical shell for axisymmetric load. Computer Methods in Applied Mechanics and Engineering 1997; 140:139-155.

3. Allik H, Hughes TJR. Finite element method for piezoelectric vibration. International Journal for Numerical Methods in Engineering 1970; 2:151-157.

4. Benjeddou A. Advances in piezoelectric finite element modeling of adaptive structural elements: a survey. Computers and Structures 2000; 76:347-363.

5. Yang JS. Mixed variational principles for piezoelectric elasticity. In Developments in Theoretical and Applied Mechanics, Antar B, Engels R, Prinaris AA, Moulden TH, Zanaboni O (eds), vol. XVI, University of Tennessee Space Institute: Tennessee, 1992; II.1.31-38.

6. EerNisse EP. Variational method for electroelastic vibration analysis. IEEE Transactions on Sonics and Ultrasonics 1967; SU-14:153-160.

7. Holland R, EerNisse EP. Variational evaluation of admittances of multielectroded three-dimensional piezoelectric structures. IEEE Transactions on Sonics and Ultrasonics 1968; SU-15:119-132.

8. Cannarozzi AA, Ubertini F. Some hybrid variational methods for linear electroelasticity problems. International Journal of Solids and Structures 2001; 38:2573-2596.

9. Sze KY, Yang X-M, Yao L-Q. Stabilized plane and axisymmetric piezoelectric finite element models. Finite Elements in Analysis and Design 2004; 40:1105-1122.

10. Wu CC, Sze KY, Huang YQ. Numerical solutions on fracture of piezoelectric materials by hybrid element. International Journal of Solids and Structures 2001; 38:4315-4329.

11. Sze KY, Yao LQ, Yi S. A hybrid stress ANS solid-shell element and its generalization for smart structure modelling. Part II-smart structure modelling. International Journal for Numerical Methods in Engineering 2000; 48:565-582.

12. Sze KY, Yao LQ. Modelling smart structures with segmented piezoelectric sensors and actuators. Journal of Sound and Vibration 2000; 235:495-520.

13. Sze KY, Pan YS. Hybrid finite element models for piezoelectric materials. Journal of Sound and Vibration 1999; 226:519-547.

14. Allman DJ. A compatible triangular element including vertex rotations for plane elasticity analysis. Computers and Structures 1984; 19:1-8.

15. Bergan PG, Felippa CA. A triangular membrane element with rotational degrees of freedom. Computer Methods in Applied Mechanics and Engineering 1985; 50:25-69.

16. Frey F. Shell finite elements with six degrees of freedom per node. ASME Winter Annual Meeting, San Francisco, 1989.

17. Jetteur P. A shallow shell element with in-plane rotational degrees of freedom. IREM Internal Report 86/3, Ecole Polytechnique Fédérale de Lausanne, 1986.

18. Jaamei S. 'Jet' thin shell finite element with drilling rotations. IREM Internal Report 88/7, Ecole Polytechnique Fédérale de Lausanne, 1988.

19. Jetteur P. Improvement of the quadrilateral 'JET' shell element for a particular class of shell problems. IREM Internal Report 87/1, Ecole Polytechnique Fédérale de Lausanne, 1987.

20. Taylor RL. Finite element analysis of linear shell problems. In The Mathematics of Finite Elements and Applications VI, MAFELAP, Whiteman JR (ed.). Academic Press Limited: London, 1987; 191-203.

21. Taylor RL, Simo JC. Bending and membrane elements for analysis of thick and thin shells. In Proceedings of the NUMETA 85 Conference, Pande GN, Middleton J (eds), Swansea, 7-11 January 1985.

22. Simo JC, Fox DD, Rifai MS. On a stress resultant geometrically exact shell model. Part II: the linear theory; computational aspects. Computer Methods in Applied Mechanics and Engineering 1989; 73:53-92.

23. Hughes TJR, Brezzi F. On drilling degrees of freedom. Computer Methods in Applied Mechanics and Engineering 1989; 72:105-121.

24. Reissner E. A note on variational principles in elasticity. International Journal of Solids and Structures $1965 ; 1: 93-95$.

25. Hughes TJR, Brezzi F, Masud A, Harari I. Finite element with drilling degree of freedom: theory and numerical evaluation. In Proceedings of the Fifth International Symposium on Numerical Methods in Engineering, Gruber R, Periaux J, Shaw RP (eds), vol. 1. Springer: Berlin, 1989; 3-17.

26. Ibrahimbegovic A, Taylor RL, Wilson EL. A robust quadrilateral membrane finite element with drilling degrees of freedom. International Journal for Numerical Methods in Engineering 1990; 30:445-457.

27. Ibrahimbegovic A, Wilson EL. A unified formulation for triangular and quadrilateral flat shell finite elements with six nodal degrees of freedom. Communications in Applied Numerical Methods 1991; 7:1-9. 
28. Sze KY, Wanji C, Cheung YK. An efficient quadrilateral plane element with drilling degrees of freedom using orthogonal stress modes. Computers and Structures 1992; 42:695-705.

29. Sze KY, Ghali A. Hybrid plane quadrilateral element with corner rotations. ASCE Journal of Structural Engineering 1993; 119:2552-2572.

30. Groenwold AA, Stander N. An efficient 4-node 24 d.o.f. thick shell finite element with 5-point quadrature. Engineering Computations 1995; 12:723-748.

31. Groenwold AA, Stander N. A 24 d.o.f. 4-node flat shell finite element for general unsymmetric orthotropic layered composites. Engineering Computations 1998; 15:518-543.

32. Dovey HH. Extension of three dimensional analysis to shell structures using the finite element idealization. Ph.D. Thesis, University of California, Berkeley, 1974, Report No. UC SESM 74-2.

33. Geyer S, Groenwold AA. Two hybrid stress membrane finite element families with drilling rotations. International Journal for Numerical Methods in Engineering 2002; 53:583-601.

34. Long CS, Loveday PW, Groenwold AA. Design of a piezoelectric mirror scanning device using topology optimization. Proceedings of the Sixth World Congress on Structural and Multidisciplinary Optimization, Rio de Janeiro, Brazil, May 2005, Paper No. 4031.

35. Pimpinelli G. An assumed strain quadrilateral element with drilling degrees of freedom. Finite Elements in Analysis and Design 2004; 41:267-283.

36. Ikeda T. Fundamentals of Piezoelectricity. Oxford University Press: Oxford, 1996.

37. Long CS. Refined piezoelectric and electroelastic finite elements with applications in topology optimization. Technical Report 2005/T/01, Structural Optimization Research Group, Department of Mechanical Engineering, University of Pretoria, 2005. (Draft Ph.D. Thesis).

38. Simo JC, Rifai MS. A class of mixed assumed strain methods and the method of incompatible modes. International Journal for Numerical Methods in Engineering 1990; 29:1595-1638.

39. Ibrahimbegovic A, Frey F. Geometrically non-linear method of incompatible modes in application to finite elasticity with independent rotations. International Journal for Numerical Methods in Engineering 1993; 36:4185-4200.

40. Liu M. Finite element analysis for cracked piezoelectric material. Master's Thesis, Department of Modern Mechanics, University of Science and Technology of China, Hefei, 1998.

41. Di S, Ramm E. On alternative hybrid stress 2D and 3D elements. Engineering Computations 1994; 11:49-68.

42. Hughes TJR, Masud A, Harari I. Numerical assessment of some membrane elements with drilling degrees of freedom. Computers and Structures 1995; 55:297-314.

43. Long CS, Geyer S, Groenwold AA. A numerical study of the effect of penalty parameters for membrane elements with independent rotation fields and penalized equilibrium, 2005, submitted.

44. Geyer S, Groenwold AA. On reduced integration and locking of flat shell finite elements with drilling rotations. Communications in Numerical Methods in Engineering 2003; 19:85-97.

45. Hughes TJR, Masud A, Harari I. Dynamic analysis and drilling degrees of freedom. International Journal for Numerical Methods in Engineering 1995; 38:3193-3210.

46. Tzou HS. Piezoelectric Shells: Distributed Sensing and Control of Continua. Kluwer Academic Publishers: Netherlands, 1993.

47. Franco Correia VM, Aguiar Gomes MA, Suleman A, Mota Soares CM, Mota Soares CA. Modelling and design of adaptive composite structures. Computer Methods in Applied Mechanics and Engineering 2000; 185:325-346.

48. MacNeal RH, Harder RL. A proposed standard set of problems to test finite element accuracy. Finite Elements in Analysis and Design 1985; 1:3-20. 